\title{
LE CODE-SWITCHING COMME RESSOURCE POUR L'ORGANISATION DE LA PAROLE-EN-INTERACTION
}

\author{
Lorenza Mondada
}

\author{
Laboratoire ICAR (CNRS) \& Université de Lyon
}

\section{Introduction}

Cet article se propose d'expliciter la contribution de la linguistique interactionnelle à l'analyse du code-switching. Pour ce faire, il passe en revue un certain nombre de recherches contemporaines sur le code-switching, en montrant qu'elles soulèvent des problèmes centraux pour la linguistique concernant notamment la théorisation de la langue, du locuteur, de la communauté, du sens, du contexte. Il synthétise les solutions proposées dans une approche interactionnelle qui considère le code-switching comme une ressource mobilisée par les participants de manière contingente, localement située, sensible à l'organisation séquentielle de l'interaction en cours. Le code-switching comme ressource endogène pour l'interaction est susceptible en effet d'éclairer aussi bien l'organisation de l'interaction que le fonctionnement de la langue comme pratique sociale.

Les phénomènes caractéristiques du parler bilingue, où les locuteurs sont susceptibles d'utiliser les ressources de plusieurs systèmes linguistiques et d'alterner entre les uns et les autres au cours de la même conversation, ont attiré l'attention des linguistes depuis la moitié du siècle dernier. C'est en effet dans les années '50 que des auteurs comme Jakobson, Vogt, Haugen et Weinreich commencent à s'intéresser à cette manifestation particulière du contact de langues qu'est le codeswitching (voir Alvarez-Caccamo (1998) pour une esquisse d'histoire critique). Depuis, la notion a subi des redéfinitions importantes, aussi bien dans son approche théorique que dans le traitement de ses évidences empiriques.

Notre point de départ sera ici la prise en compte du code-switching comme d'un laboratoire exemplaire pour la linguistique, un terrain où ses modèles théoriques et analytiques sont confrontés à des phénomènes interrogeant de manière radicale leurs présupposés et leurs capacités explicatives. Après avoir identifié un certain nombre d'enjeux ainsi posés par le code-switching à la linguistique (1.), nous allons nous concentrer sur une question particulière, bien que centrale : les explications des fonctionnalités pragmatiques et socio-linguistiques du code-switching (2.). Nous le ferons en passant en revue différentes réponses données au problème de la polyfonctionnalité du codeswitching, au sein de modèles qui soit essaient de la réduire en cherchant à identifier une distribution fonctionnelle des langues selon les activités sociales, soit reconnaissent la pluralité des fonctions que peut revêtir leur alternance. La solution proposée par la linguistique interactionnelle 
inspirée de l'analyse conversationnelle sera le point de départ pour une reconceptualisation des problèmes présentés en introduction (3.). Cela nous permettra à la fois de revisiter la question de la langue en interaction et de montrer la manière dont le code-switching est une ressource puissante pour l'organisation de l'interaction elle-même (4.).

\subsection{Quelques défis posés par le code-switching à la linguistique}

Le phénomène du code-switching (désormais CS), dans lequel des interlocuteurs alternent d'une langue à une autre au cours de la même interaction, pose une série de défis théoriques aux modèles linguistiques. Nous allons les inventorier ici, pour en reproposer plus loin (3.) une reformulation au sein de la linguistique interactionnelle.

1. Alors que les modèles de la grammaire sont plus ou moins tacitement basés sur une seule langue, considérée comme un système homogène, le CS pose le problème de la prise en compte non seulement de plusieurs variétés mais encore de plusieurs langues au sein du même échange (CS dit « inter-phrastique »), voire au sein du même énoncé (CS «intra-phrastique »). Cette pluralité de langues en contact pose d'autant plus de problèmes que celles-ci présentent dans certains cas des processus d'hybridation (relevant du continuum allant du code-mixing, au language mixing, voire aux fused lects, où les langues sont confondues, où il est difficile d'identifier la langue " matrice » et où on ne peut plus attribuer des fonctionnalités à des formes distinctes ${ }^{1}$ ). L'intégrité et l'homogénéité du système linguistique sont donc être mises à mal par le CS.

2. Au-delà des problèmes posés par l'homogénéité des systèmes linguistiques, les phénomènes de code-switchng questionnent la conception même du « système »- et d'ailleurs la référence à une alternance de «codes» dans la dénomination même du phénomène pose problème (cf. infra 3.1.). Plutôt qu'un «système" ou qu'un «code», nous avons ici affaire à un ensemble mouvant de ressources bricolées en temps réel par les locuteurs. Cela invite à concevoir la grammaire moins comme un système que comme une pratique sociale.

3. Les phénomènes du CS ont longtemps été traités comme relevant d'un manque de compétence ; puis, au contraire, comme la manifestation d'une compétence bilingue sophistiquée. Dans les deux cas, ils montrent combien cette pratique bilingue questionne la notion de « locuteur idéal » sous-jacente à de nombreux modèles linguistiques, et dont on trouve la transposition dans la notion de «bilingue idéal» régissant la définition du bilinguisme de Bloomfield comme un «native-like control of two languages» (Bloomfield 1933: 55). Ces références montrent que le modèle du «locuteur idéal » est celui d'un «locuteur natif» idéalisé, qui a été mis à mal dans des controverses récentes, critiquant la référence à la «nativité » (Kandiah 1998, Davies 2003, Renaud 1998) et critiquant l'impérialisme voire le colonialisme sous-jacent à cette notion (le locuteur natif étant dans le cas de l'Anglais d'origine britannique mais jamais d'origine indienne ou singapourienne). De même, la relativisation de la maîtrise parfaite des différentes langues chez le bilingue et la reconnaissance des apprenants de L2 voire de L3 comme locuteurs bilingues a porté à une mise en cause de la notion de « langue maternelle » (Lüdi \& Py $2003: 44$ sv).

4. Si l'on passe maintenant du système et du locuteur vers les usages pratiques du CS, ceux-ci posent le problème de savoir quand et pourquoi les locuteurs alternent entre une langue et l'autre. Comment interpréter le surplus de sens que produit le CS, son rendement pragmatique ? Cela repose une question fondamentale en linguistique, concernant l'articulation entre « forme » et « fonction»,

\footnotetext{
${ }^{1}$ Les typologies et définitions de ces phénomènes subissent des variations importantes selon les auteurs et nous n'en visons pas ici une synthèse. Voir Auer (1998a) pour des considérations intéressantes sur le passage du code-switching au mixed-language puis à sa stabilisation en fused lects.
} 
concernant la fonctionnalité de la variation et des choix de langue. Plus précisément, cela pose la question de l'articulation entre mobilisation des ressources grammaticales et organisation de la parole en interaction.

5. Le CS a été depuis toujours rapporté à des contextes socio-culturels caractérisés par le contact entre différentes langues, par la mobilité des locuteurs, par des enjeux identitaires et sociaux exprimés notamment à travers les pratiques langagières. Il repose ainsi de manière plus générale la question de l'articulation entre langue, société, culture, contexte. Comme dans d'autres domaines de la linguistique, plusieurs modèles sont disponibles pour penser ces articulations: ils peuvent privilégier une interprétation spéculaire du CS comme reflet (des structures sociales, des idéologies, des identités, des appartenances culturelles ou etniques, des conflits, des rapports de dominance et de pouvoir, etc.) ; ils peuvent lui préférer une interprétation corrélationniste et distributionnelle associant un certain type d'actes de langage ou d'activité à une variété (le discours privé vs public, la sélection d'un interlocuteur, la négociation de requêtes, l'organisation du discours rapporté, la gestion du désaccord, l'expression de l'humour ou de l'affect, la manifestation d'une position énonciative, etc.). Nous montrerons dans ce qui suit que si modèles peuvent rendre compte de tendances générales, ils rendent plus difficilement compte du détail des CSs observables dans la parole-en-interaction et que, pour ce faire, plutôt que de partir d'une liste - nécessairement ouverte - de fonctions, il vaut mieux adopter un modèle séquentiel de la manière dont les participants euxmêmes organisent et rendent sensé le CS de manière contingente et occasionnée au fil de l'interaction.

\section{Rendre compte des fonctionnalités socio-pragmatiques du code-switching}

Nous allons reprendre les deux dernières questions posées ci-dessus pour développer la manière dont différents modèles du CS ont formulé l'articulation entre les choix de langue et leurs rendements fonctionnels. Cela nous permettra de poser plus généralement la question du lien entre forme et fonction, entre grammaire et interaction.

\subsection{A la recherche de spécialisations fonctionnelles des choix de langue}

La question des rendements fonctionnels du choix de langue a été posée en s'orientant vers différentes solutions possibles : d'une part, on trouve des tentatives pour spécifier le mieux possible l'articulation entre une forme (un code) et une fonction spécifique, de manière à limiter les intersections entre sphères d'usage différentes des mêmes ressources. D'autre part, on trouve des modèles qui admettent la polyfonctionnalité des choix formels, tout en ayant des difficultés à les expliquer de manière exhaustive.

Les premiers modèles qui se sont préoccupés de rendre compte de la fonctionnalité des choix de langues dans des sociétés bilingues ont privilégié une approche macro en prenant en compte moins des locuteurs individuels que des communautés entières, caractérisées par des pratiques bilingues historiquement stabilisées. Il s'agit de tentatives, sans doute inspirées par la tradition de l'Etat nation (Heller 1992, 1995; Blommaert \& Verschueren 1998), de faire coïncider les limites territoriales, ethniques-culturelles (communautaires) et linguistiques et de faire correspondre à une forme une fonction, une valeur unique, selon une distribution et une spécialisation fonctionnelles strictes.

Ainsi, la notion de « diglossie » permet à Ferguson (1959) de rendre compte des fonctions que pouvaient avoir des variétés différentes dans une communauté, en distinguant une variété haute (High) et une ou plusieurs variétés basses (Low). Ferguson postule une spécialisation fonctionnelle de $\mathrm{H}$ et $\mathrm{L}$ : dans un certain nombre de situations, seule la variété $\mathrm{H}$ est appropriée, dotée de prestige, de valeur patrimoniale, de stabilité, de standardisation, et expression de la parole publique ; dans 
d'autres, c'est L, la variété vernaculaire, souvent orale, utilisée dans des contextes informels, expression de l'intimité (pour une discussion voir Lüdi \& Py, 2003 : 11-17) : la superposition entre les deux est réduite. Ce modèle a permis de rendre compte d'un certain nombre de cas historiques (les exemples données par Ferguson sont le Créole et le Français en Haiti, le Suisse Allemand et l'Allemand Standard en Suisse, l'Arabe classique et l'Arabe courant en Egypte), dans une description où la séparation entre $\mathrm{H}$ et $\mathrm{L}$ tend à exclure le CS par une spécialisation des contextes d'usage de chaque langue. Fishman va élargir cette problématique initiale en ajoutant à la diglossie sociétale (émanation d'une collectivité, voire d'un Etat) le bilinguisme individuel (1967). En se focalisant sur la description ce dernier, il reprend l'idée de Weinreich (Weinreich 1968:73) que les locuteurs switchent selon le topic, l'interlocuteur ou l'activité en cours. Il élabore ainsi une différenciation des variétés linguistiques utilisées par «domaines » (pour une discussion critique, voir Renaud 2005), d'une manière qui assigne normativement (i.e. lorsque l'usage est « approprié ») une variété à un domaine: " 'Proper' usage, or common usage, or both, dictate that only one of the theoretically co-available languages will be chosen by particular classes of interlocutors on particular occasions » (1965: 67, italique dans l'original). Le domaine (p.ex. la famille, le travail, les amis, l'école, l'administration...) permet d'articuler des choix de langue relatifs au topic - propres aux interactions individuelles - avec des normes socio-culturelles plus larges ; de même, il permet d'articuler l'identité des locuteurs avec des rôles propres à des contextes institutionnels. En outre, Fishman admet des sources de variabilité au sein de chaque domaine (relatives au média employé, écrit ou parlé, en production ou en compréhension, et au degré de formalité).

Même si Fishman opère le passage d'un point de vue macro, centré sur la communauté dans son ensemble, vers un point de vue individuel, il continue à traiter les codes en présence comme des systèmes de formes et de valeurs délimités, ne se chevauchant que minimalement, distribués de manière mutuellement exclusive dans différentes arènes et activités sociales, permettant ainsi de rendre compte d'une distribution complémentaire des pratiques multilingues dans une société donnée. Cette distribution tend à réduire fortement les phénomènes de CS, puisque l'emploi de chaque variété est spécialisé pour un domaine d'activité spécifique.

\subsection{Le code-switching comme indexation conventionnalisée de droits et obligations}

La question de la valeur fonctionnelle du CS a aussi été posée dans le cadre des approches qui ont privilégié son explication par les contraintes grammaticales qui s'exercent sur lui. Dans cette perspective, les contraintes formelles sont premières et priment sur les fonctionnalités pragmatiques: Myers-Scotton parle d'une «division of labour which gives structural factors the role of prime mover : First, grammatical processes designate permissible forms of CS; then social processes regulate selection among the range of permissible forms. » (1993a : 476). Toutefois, les choix effectués par les locuteurs au sein des contraintes grammaticales et leurs motivations sociales sont importants pour la suppression ou le développement de certaines options formelles, et donc pour le changement linguistique.

Plusieurs modèles des contraintes grammaticales ont été formulés.

Poplack (1980) établit une hiérarchie des fréquences des constituants alternables et montre que les alternances tendent à avoir lieu aux frontières de constituants (entre deux énoncés, entre NP et VP par exemple). Son modèle se base sur l'étude des alternances entre espagnol et anglais dans la communauté portoricaine et se fonde sur deux principes expliquant le CS inter-phrastique et intraphrastique :

l'alternance n'a pas lieu entre un morphème lié et une forme lexicale tant que cette dernière n'a pas été intégrée morphologiquement dans la langue du premier (free morpheme constraint); 
- l'alternance tend à avoir lieu là où elle ne viole la syntaxe d'aucune des langues en présence, en privilégiant donc des frontières de constituants similaires dans les deux langues (equivalence constraint).

Le premier principe ne semble pas fonctionner pour les données recueillies en Afrique de l'Est par Myers-Scotton $(1988: 157,1993 b)$ qui, pour rendre compte des phénomènes présents dans ses corpus, élabore le Matrix Language Frame Model, qui rend compte du CS intraphrastique en distinguant entre matrix language et embedded language. Le premier fournit le cadre syntaxique de l'énoncé dans lequel s'intègre et auquel s'ajuste le second. Le premier jouit d'un statut sociolinguistique distinct du second, est généralement mieux maîtrisé par le locuteur et est le plus fréquemment utilisé dans les énoncés alternés.

Ce modèle s'articule au Markedness Model qui rend compte des motivations sociales du CS : étant donnée une situation où se déroule un certain type d'interaction, les locuteurs traitent un certain code comme normativement et conventionnellement attendu (non-marqué) dans ce contexte, en tant qu'indexant un ensemble de droits et obligations: en utilisant le code non-marqué, ils adhèrent aux normes en question et maintiennent un statu quo des positions. Autrement dit, "all speakers have mental representations of a matching between code choices and rights and obligations sets" (Myers-Scotton 1988 : 152). Le modèle propose donc une motivation de la distribution typique des choix de codes dans un type conventionnalisé d'échange donné. Lorsque le choix est marqué, le locuteur entend renégocier sa position par rapport à celle qui était attendue.

Le CS intraphrastique tend à être un CS non-marqué davantage qu'un CS marqué ; dans ce cas, le choix de la langue matrice constitue le choix des normes sociales auxquelles le locuteur adhère dans son CS non-marqué.

Ce modèle suppose que les locuteurs puissent associer toute interaction dans laquelle ils s'engagent à un type d'échange doté d'attentes normatives précises; cela suppose pareillement que les échanges soient régis par des normes conventionnelles définissant des ensembles de droits et obligations (" a conventionalized exchange is any interaction for which speech community members have a sense of 'script'» 1988: 152). Si l'échange n'est pas conventionnalisé, les choix linguistiques seront exploratoires, les locuteurs proposant des candidats à devenir des index d'une relation mutuellement acceptée (1988: 155). Dans ce sens, le modèle se veut sensible aux variations contextuelles et à la possibilité que les participants renégocient l'ensemble des droits et obligations qu'ils avaient adoptés précédemment. Par exemple, l'interaction avec les agents de sécurité à l'entrée d'un centre d'affaires à Nairobi constitue un échange conventionnalisé dans cette communauté, où les traits saillants concernent l'apparence du visiteur (s'il est identifié comme un Africain, le choix non marqué privilégie le Swahili, si une appartenance ethnique commune est relevée, en revanche, le choix non marqué préférera la langue de l'ethnie): la description ethnographique de l'observateur, ainsi que sa catégorisation des locuteurs et des éléments saillants du contexte joue donc un rôle central dans l'analyse de la manière dont le CS « indexe » ces traits.

La notion d'«indexicalité» renvoie ici moins à une dépendance contextuelle et à une construction contingente au contexte qu'à une association plus ou moins stable, plus ou moins conventionnalisée, entre des types d'interaction (identifiés comme tels par le savoir sociolinguistique des locuteurs), des ensembles de droits et obligations les caractérisant et des choix de formes adoptant rationnellement un ensemble particulier de ces droits et obligations. Ainsi par exemple (Myers-Scotton \& Bolonyai 2001 : 9) un repas de famille hongrois auquel participe un membre de la famille émigré aux USA est un type d'interaction caractérisé par un ensemble de droits et obligations non marqués préférant un choix linguistique unique, voulant que tout le monde parle hongrois à la maison. Un autre ensemble de droits et obligations, distancé du premier, marqué, est indexé par une conduite qui consisterait à parler systématiquement anglais. Un ensemble intermédiaire, manifestant un compromis, est constitué par le CS entre hongrois et anglais. Le choix 
linguistique est relié ici moins à la dynamique interactionnelle (p.ex. à des positions séquentielles particulières, où une action particulière serait implémentée en réponse à une autre action) qu'à une définition pré-existante de normes familiales (plus généralement, des facteurs saillants de la communauté et du type d'interaction) auxquelles les participants peuvent s'adapter ou non (pour une critique sociolinguistique voir Meeuwis \& Blommaert 1994).

Plus récemment, le modèle s'est enrichi d'une composante appelée modèle du choix rationnel (Rational Choice Model) (Myers-Scotton \& Bolonyai 2001), soulignant la dimension «calculée » des décisions des locuteurs bilingues, tenant compte de coûts et bénéfices dans le choix de l'action la mieux adaptée à leurs intentions. Toutefois le modèle ne traite pas le processus par lequel cette rationalité postulée met en forme une action particulière parmi d'autres - sauf à postuler une cohérence entre les deux et une relation transparente entre cette action et les intentions qui l'ont motivée (Li Wei 2005 : 377)

\subsection{Le problème de la prolifération des fonctions}

Dès que l'on passe d'une approche basée sur la distribution conventionnalisée d'activités, de normes, de valeurs dans une communauté à une approche basée sur les conduites observables des locuteurs bilingues au sein de contextes d'activités particuliers, il devient souvent difficile de modéliser de manière exhaustive les fonctions que revêt le CS dans les cas particuliers observés. C'est pourquoi dans la littérature la réponse à la question de savoir quel est le sens produit par le CS est souvent formulée sous forme de listes ouvertes de fonctions.

Le CS est ainsi considéré comme permettant (voir p.ex. Alvarez-Caccamo 1990 ; Lüdi \& Py, $2003: 153 \mathrm{sv}$, reprenant Grosjean 1982:152sv) de résoudre une difficulté d'accès au lexique ou une recherche de mots, d'exhiber son appartenance ou celle des partenaires à la communauté bilingue, de sélectionner un destinataire particulier, de distribuer des rôles aux participants associés à leurs langues, de gérer les topics et organiser l'avant- et l'arrière-plan topical, d'exprimer une polyphonie de voix, notamment dans les discours cités, de structurer des récits ou d'autres discours complexes, d'exprimer une fonction métacommunicative en suggérant une certaine interprétation de l'énoncé, d'accroître le potentiel référentiel du lexique, d'exploiter les spécificités d'expressions dans une langue particulière, etc..

Ces listes de fonctions présentent certes l'avantage d'être issues d'observations empiriques et d'offrir des pistes pour analyser des occurrences attestées, mais posent néanmoins plusieurs problèmes : a) elles sont souvent ouvertes, ne pouvant prétendre à l'exhaustivité ; b) elles réunissent des fonctionnalités hétérogènes, certaines relevant des processus cognitifs, d'autres de pratiques sociales, d'autres encore de fonctions discursives - présupposant souvent des modèles très différents du CS lui-même; c) elles confondent souvent les formes utilisées et les effets qu'elles produisent.

Plus radicalement, elles posent le problème de la conceptualisation de l'association (selon un modèle de pure corrélation? selon un modèle causal ou spéculaire ? selon un modèle intentionnel et rationnel ?) entre le CS et les différentes entités censées l'expliquer.

D'une part, associer directement le CS à des entités « extra-linguistiques » telles que l'identité, les valeurs d'appartenance, le statut social, etc. produit un modèle très rigide (même lorsqu'il prévoit que ces relations puissent être renégociées) basé sur une relation directe entre p.ex. appartenance ethnique et choix de langue. D'autre part, associer directement le CS à une activité ou à un acte de langage homogénéise des domaines d'activité et en réifie les frontières d'une manière qui n'est pas démontrable sur la base de données empiriques attestées - à l'exception peut-être de rituels extrêmement stables et formalisés, associant de manière stricte une activité et un registre langagier - : les corpus d'interactions montrent dans leur détail une organisation beaucoup plus complexe des activités elles-mêmes et une pluralité beaucoup plus vaste de ressources mobilisées pour les structurer et leur donner du sens. En outre, ces associations ne prennent pas en 
considération le contexte, dans le double sens de la situation sociale et de l'environnement séquentiel, où le CS est observable.

\subsection{Le code-switching comme indice de contextualisation}

Une solution permettant de formuler des principes généraux agissant à la base de la pragmatique du CS consiste à le traiter en tant qu'indice de contextualisation, selon la terminologie de Gumperz (1982).

Avant de formuler cette solution, Gumperz a travaillé sur le CS en Inde puis en Norvège, avec son collègue J. Blom. C'est dans la petite ville de Hemnesberget que Blom et Gumperz remarquent que les alternances de codes sont systématiques et prédictibles. Pour en rendre compte, ils distinguent entre code-switching transactionnel (ou situationnel) et code-switching métaphorique. Le premier rend compte de l'alternance lorsqu'il y a changement de situation, d'activité, d'interlocuteur, i.e. un changement intervenant dans le contexte. Par exemple les enseignants donnent leurs cours dans le dialecte standard (Bokmål) mais passent au dialecte local (Ranamål) dans des activités où ils encouragent les élèves à la discussion (Blom \& Gumperz 1972 : 424). Le second n'est pas sensible à un changement de situation ou d'interlocuteurs, ceux-ci restant les mêmes, mais exploite l'alternance pour viser des effets communicationnels, comme distinguer le discours citant et le discours cité, la partie thématique et la partie rhématique, la version normale et la version emphatisée d'un même énoncé répété dans l'autre langue, voire un engagement personnel vs une distanciation, etc. Par exemple, dans les bureaux de l'administration locale, clercs et clients échangent des salutations et des nouvelles en Ranamål, alors qu'ils passent au Bokmål pour parler affaires (Blom \& Gumperz, 1972 : 425).

Plus tard, Gumperz reconceptualise le CS à l'aide de la notion d'indice de contextualisation (contextualisation cue), en adoptant une perspective plus abstraite et plus générale sur le CS comme contribuant à la construction du sens de ce qui est énoncé. De manière générale, les indices de contextualisation sont des pratiques par lesquelles les participants mettent en évidence, reproduisent, transforment, effacent des éléments du contexte qu'ils rendent pertinents pour l'interprétation de l'énoncé en cours. Le CS est l'une des ressources qui accomplissent ce travail de guidage interprétatif, notamment en créant un contraste entre un segment énoncé dans une langue et le segment suivant énoncé dans l'autre : ils soulignent ainsi une transformation dans les détails à prendre en compte pour l'interprétation.

Les indices de contextualisation ont les propriétés suivantes (Gumperz 1982, ch. 3) :

- $\quad$ ils exploitent des éléments de la langue qui n'ont pas une valeur référentielle propre, comme la prosodie, le CS, le style-shift, les gestes, etc.;

- ils sont souvent multiples et convergents, présentant une certaine redondance, notamment à travers les différentes modalités (du linguistique au prosodique au kinésique) ;

- ils acquièrent un sens grâce à des processus inférentiels situés, et non par association conventionnelle et fixe entre une forme (l'indice) et une fonction déterminée ;

- leur sens échappe souvent à la conscience métalinguistique et est constitué par un ensemble holiste d'assomptions d'arrière-plan, intervenant dans l'interprétation des énoncés (cela expliquant le fait qu'ils ne font pas l'objet d'un apprentissage explicite, étant plutôt acquis par immersion et exposition, et qu'ils sont souvent à la base de malentendus, notamment en situation interculturelle) ; 
- leur sens est organisé par un contraste entre ce qui précède et ce qui est contextualisé, donc détaché, mis en évidence, transformé par une rupture provoquée par l'indice de contextualisation.

- ils pointent vers un certain élément du contexte qu'ils rendent pertinent pour l'interprétation de l'activité en cours; ce faisant ils exploitent et ils constituent ce contexte: "Rather than claiming that speakers use language in response to a fixed, predetermined set of prescriptions, it seems more reasonable to assume that they built their own and their audience's abstract understanding of situational norms, to communicate metaphoric information about how they intend their words to be understood» (Gumperz 1982 : 61). Le contexte est ainsi imbriqué dans les ressources linguistiques, qui le configurent en pointant vers un aspect particulier rendu pertinent. La différence de taille introduite par Gumperz par rapport aux analyse fonctionnelles de Fishman est que le contexte est ici activement construit par les participants, dans le formatage de leurs productions langagières, pouvant utiliser le CS autant pour s'y ajuster que pour l'instituer - en ne se limitant donc pas simplement à s'accommoder à une série de prescriptions extérieures (Auer \& di Luzio 1992).

\subsection{Le code-switching comme ressource interactionnelle organisée séquentiellement}

Le travail de Gumperz a influencé durablement les recherches sur le CS dans l'interaction, en attirant l'attention sur les ressources mises en oeuvre par les participants eux-mêmes pour signaler telle ou telle valeur contextuelle de leur parole. Ces apports ont été repris par Peter Auer depuis les années ' 80 , au sein d'une approche conversationnelle du CS, strictement basée sur l'organisation séquentielle de l'interaction. Dans ce cadre, le CS est abordé en tant que coexistence de ressources provenant de systèmes différents, reconnus comme tels par les participants et traités par eux dans une relation de contiguité, i.e. dans une relation de previous et de next (ce qui exclut la prise en compte de fragments en langues différentes tirés de moments conversationnels distincts). Cette approche permet de reprendre une dimension importante du CS comme indice de contextualisation : c'est en vertu du contraste qui est produit entre un ensemble de ressources et un autre qui lui succède que les participants construisent le sens du CS. Ce contraste reçoit une interprétation en fonction de son placement séquentiel - en réponse à la question qui soutient constamment l'activité interactionnelle des co-participants : «why that now?».

Auer $(1984,1988,1995,1998 b)$ croise deux dimensions dans son approche du CS :

- il s'inspire de Gumperz pour établir une différence entre participant-related $C S$ et discourse-related CS: le premier s'oriente vers les participants et leur attribue des compétences et des préférences linguistiques auxquelles il s'ajuste; le second signale un changement dans l'organisation de l'interaction, relatif à la constellation des participants, au mode d'interaction, à l'enchaînement séquentiel, à l'action en cours, à l'initiation d'une nouvelle séquence ou activité, etc.

— il définit une série de patterns séquentiels caractérisant l'alternance des ressources (dans la représentation schématique qui suit, A et B sont les langues co-présentes et 1 et 2 sont les locuteurs) :

- alternance de langue: alors que la langue A a été établie comme langue de l'interaction, se produit, soit entre un tour et un autre, soit au sein d'un même tour, une alternance qui fait que l'interaction se poursuivra dans cette nouvelle langue :

A1 A2 A1 A2//B1 B2 B1 B2 ou bien A1 A2 A1//B1 B2 B1 B2 
Cette alternance est principalement relative au discours.

- négociation de langue: le locuteur 1 utilise la langue A alors que le locuteur B utilise la langue 2 ; il est possible qu'après un certain temps un des deux se rallie à la langue de l'autre :

A1 B2 A1 B2 A1 B2 ou bien A1 B2 A1 B2 A1//A2 A1 A2

Ce pattern est souvent lié aux préférences des participants (il est donc participant-related) pour une langue ou pour une autre, qui peuvent être dues à des questions de compétence mais aussi à des questions d'idéologie ou de manifestation d'appartenance.

- choix de langue laissé ouvert: les locuteurs switchent au sein de leurs tours et non seulement entre les tours, cela pouvant être accompli par tous les locuteurs ou bien par un seul, l'autre continuant à manifester sa préférence :

\section{AB1 AB2 AB1 AB2 ou bien AB1//A2 A1 A2}

- transfert (utilisé indépendamment de la définition du «transfer» qui sert à décrire les variétés de contact et reformulé ensuite par le terme d'insertion): contrairement aux cas d'alternance, traités précédemment, où on ne sait pas a priori quand le segment dans une langue donnée va se terminer et où il n'est parfois pas possible de déterminer quelle est la langue de base de l'interaction, le changement de langue qui se produit en insertion a lieu au sein d'u n tour et n'affecte pas la langue de l'interaction, puisqu'il y a ensuite un retour à la langue initiale (mais voir Auer 1995, 17sv sur la difficulté de distinguer entre alternance et insertion dans certains cas) :

\section{$\mathrm{A} 1[\mathrm{~B} 1] \mathrm{A} 1$}

Depuis la contribution essentielle de Auer, plusieurs travaux ont proposé une analyse conversationnelle du CS, en se basant sur la «mentalité analytique » développée par ce courant (Cromdal 2000 ; Garafanga 2001, 2005 ; Steensig 2003 ; Wei 2002, Mondada 2004, Mondada sous presse). Dans ce cadre, ce qui prime ce n'est pas la question de savoir "pourquoi » les locuteurs opèrent les choix qu'ils font mais d'abord la description du «comment» de leurs pratiques sociales. La focalisation sur le «comment» de l'action découle de l'attention portée aux détails de l'interaction, conçus non comme des «marqueurs » dotés de fonctions prédéfinies par le modèle de l'analyste, mais d'abord comme des ressources endogènes pertinentes pour les participants, mises en oeuvre par et pour eux, dans la production reconnaissable et intelligible de leur conduite, dont ils sont les premiers interprètes. C'est ainsi que l'analyse conversationnelle se penche sur le CS tel qu'il est exploité au sein des "méthodes » (au sens garfinkelien) par lesquelles les participants formatent leurs tours, coordonnent leurs actions, initient des séquences et s'alignent ou non par rapport à ce qui a été projeté dans l'action précédente. L'action est donc étudiée du point de vue des enchaînements séquentiels tels qu'ils sont localement organisés par les participants, chaque tour fournissant le contexte pertinent pour le tour suivant, et non du point de vue de «motifs » extérieurs qui guideraient les interlocuteurs (et qui seraient reconstitués par l'analyste indépendamment des actions des participants) $)^{2}$.

Il en découle une approche du CS dans l'interaction qui le traite comme une ressource pour accomplir des activités interactionnelles, dont le caractère méthodique est visible pour les interactants eux-mêmes dans la prise en considération de ce qui le précède et le suit, dans les

\footnotetext{
${ }^{2}$ Voir Wei (2002: 168-169) pour un exemple de réanalyse d'une occurrence de CS dans une perspective conversationnelle d'un extrait analysé précédemment dans une perspective exogène ; voir dans le même article l'analyse d'un même extrait du point de vue du markedness model et de l'analyse conversationnelle (Wei $2002: 172-174,2005: 377$ sv).
} 
relations rétrospectives au tour ou au TCU (turn-constructional unit) précédent et dans les relations prospectives au tour ou au TCU suivant, dans le type de (dés)alignement produit relativement à ce qui précède et la projection de ce qui suit. Autrement dit, l'effet et le traitement d'un CS à un point séquentiel donné, est/sont visible(s) lorsqu'il/ce CS est pertinent pour les participants (lorsqu'il est "procedurally consequential», Schegloff 1992) dans la manière dont ils y répondent dans l'organisation de la suite qu'ils donnent à l'action - cela privilégiant l'interprétation endogène et située des participants par rapport aux explications exogènes possibles de l'analyste. Cela ne conduit pas à une vision autonome du CS en interaction qui serait détachée du contexte social ; le contexte social est lui-même considéré non comme externe à l'interaction (qui «indexerait» ou actualiserait certaines de ses composantes) mais comme étant reproduit, transformé, réélaboré de l'intérieur de l'interaction, dans son organisation même.

\section{Conséquences : redéfinition de certains enjeux}

De cette conception découlent une série de reconceptualisations de problèmes classiques qui ont été posé à propos du CS. Nous n'expliciterons que deux ensembles de problématiques, les premières concernant la question de la «langue » comme ressource située (3.1) et la seconde concernant la manière dont penser l'articulation entre CS et identités en action (3.2). Dans les deux cas, nous esquisserons quelques enjeux méthodologiques liés à la transcription des données - un point sur lequel l'analyse conversationnelle a contribué sensiblement en linguistique.

\subsection{Langue, code, ressources}

La métaphore du «code » entraîne avec elle de nombreux présupposés : les processus sous-jacents sont ceux du «codage » et du "décodage», reposant sur un "code» entendu comme une clef conventionnelle permettant d'effectuer ces opérations de manière symétrique. Cela pourrait laisser entendre qu'il y aurait des fonctions conventionnellement associées à des formes du CS et que l'on pourrait passer aisément des unes aux autres.

En outre, la métaphore du code vient renforcer une vision traditionnelle des langues comme constituant des systèmes homogènes distincts les uns des autres; il est vrai que dans certains cas, cette vision est problématisée: « any item that occurs in speech must be a part of some language if it is to convey any meaning to the hearer. [...] The real question is whether a given stretch of speech is to be assigned to one language or the other. » (Haugen $1956: 39)$. Néanmoins, la question reste ici posée du point de vue du linguiste : c'est grâce à son savoir, aux typologies linguistiques en vigueur, qu'il décide de définir tel morphème comme relevant de telle ou telle langue.

Posée au contraire du point de vue du participant, la question est de savoir si les locuteurs reconnaissent différentes langues, non de manière générale mais dans une situation conversationnelle donnée. Cela revient à dire que l'appartenance d'une forme à une langue est un accomplissement situé, contingent, dépendant de l'activité en cours et de son organisation: les locuteurs peuvent traiter à toutes fins pratiques une forme comme appartenant à une langue précise ou bien comme étant « neutre » par rapport à son appartenance (Alvarez-Caccamo 1990 ; Mondada $2000 \mathrm{~b})^{3}$. En outre, on peut se demander si les locuteurs inscrivent les formes dans des langues identifiées comme telles ou s'il ne suffit pas simplement, pour les fins pratiques de la conversation, qu'ils établissent des contrastes entre ressources, peu importe la catégorisation linguistique de l'appartenance des éléments contrastés ou peu importent les valeurs asssociées à l'une ou à l'autre

\footnotetext{
${ }^{3}$ Ainsi les locuteurs du galicien peuvent parfois traiter la différence entre castillan et galicien comme non pertinente ou au contraire comme configurante (Alvarez-Caccamo 1990); par ailleurs Alfonzetti (1998) montre que si la différence entre standard et dialecte peut parfois sembler minime aux yeux du linguiste, elle est dotée de forte contrastivité pour les locuteurs.
} 
langue ${ }^{4}$. Dès lors, la catégorisation des «codes» dans le CS est un problème pratique des participants : les catégorisations du linguiste - distinguant p.ex. entre galicien, castillan, ou des formes qui sont «neutres" par rapport aux deux premières (Alvarez-Caccamo 1990) - ne correspondent pas nécessairement à la catégorisation qu'en font les participants. De même, Sobrero parle d'une «strategia di attenuazione » qui minimise la différence entre codes, en exploitant une "zona neutra, di transizione in cui si produce un elemento [...] fortemente 'incrociato' fra i due codici in gioco, in modo tale che non lo si può assegnare nè al dialetto nè all'italiano » (1992:14). Dans d'autres cas, ils peuvent plutôt s'orienter vers du code-mixing plutôt que vers du codeswitching, i.e. vers une hybridation traitée globalement comme une langue unique et où les éléments de l'une ou de l'autre langue de départ ne sont plus distingués fonctionnellement : dans ce cas, les locuteurs n'opèrent plus de choix et d'alternances linguistiques (Auer 1995 : 15-16).

Dans ce cadre, le fait de parler de «ressources » linguistiques plutôt que d'éléments inscrits $a$ priori dans une langue, permet de rendre compte du caractère localement accompli - donc n'allant pas de soi, ne préexistant pas toujours à l'interaction, n'étant pas toujours stabilisé - de l'appartenance d'une ressource à une langue particulière. La notion de ressource permet de reconnaître que les caractéristiques formelles de l'interaction sont indexicales, contingentes, occasionnées et à toutes fins pratiques : aux fins pratiques de l'interaction, le locuteur peut les traiter comme appartenant à un répertoire normé, préexistant, identifié comme tel, mais il peut aussi les traiter comme étant des ressources plastiquement redéfinissables selon les occasions conversationnelles.

Cette conception a des conséquences importantes non seulement au plan théorique, mais aussi pour l'approche empirique des données. Celles-ci, une fois enregistrées, sont transcrites pour être soumises à l'analyse : la transcription est une pratique de production et de configuration des données secondaires (Mondada 2006) à partir des données primaires elles-mêmes « fabriquées » par les enregistrements, au fil de laquelle est effectuée une spatialisation et une textualisation de la parole-en-interaction. Cette textualisation implique des choix importants ${ }^{5}$ concernant :

- $\quad$ l'identification et la délimitation d'unités - morphèmes, lexèmes - par l'écriture,

- l'adoption de l'orthographe (standard ou aménagée) d'une langue plutôt que d'une autre,

- la codification éventuelle du texte par des choix typographiques, renvoyant eux aussi à une langue plutôt qu'à une autre (tel est le cas dans la pratique consistant à baliser le CS dans la transcription en adoptant une police, p.ex. le romain, pour une langue et une autre, p.ex. l'italique, pour une autre) ${ }^{6}$.

\footnotetext{
${ }^{4}$ Par exemple Alfonzetti (1998 : 199-202) donne des exemples de CS servant à distinguer fonctionnellement discours cité et discours citant où la langue utilisée pour le discours cité n'est surement pas la langue qui aurait pu être utilisée dans l'événement rapporté : le CS a ici une fonction de délimitation et de contraste mais pas de fonction mimétique, où la langue elle-même indexerait des valeurs particulières.

${ }^{5}$ Pour une discussion des implications théoriques des choix de transcription du parler bilingue voir Mondada (2000a) ; sur les transcriptions plurilingues voir aussi Auer (1995) et Orletti \& Testa (1991).

${ }^{6}$ Cette pratique impose un choix tranché quant à la catégorisation de l'appartenance de chaque morphème à un "code » ou à un autre, voire à la langue matrice vs à la langue insérée. Ainsi par exemple les transcriptions de Poplack (1980) dans son article classique sur CS entre anglais et espagnol, distinguent typographiquement les deux langues (alternant entre le romain et les capitales), en utilisant systématiquement le romain pour la langue de base : cela catégorise l'appartenance des morphèmes ainsi transcrits à une langue particulière et identifie cette langue comme étant la langue de base dans l'usage ainsi documenté. Cette convention n'est toutefois pas explicitée comme telle et ne fait l'objet d'aucun commentaire - alors que l'article thématise les modes de codage du corpus pour ce qui est des catégories syntaxiques ayant servi à l'annoter (1980: 596sv). Le codage typographique n'est donc souvent pas considéré comme une opération à expliciter, bien qu'il incorpore des décisions importantes.
} 
Ces choix, imposés par le dispositif même de l'écriture, ont pour effet de catégoriser l'oral, d'identifier des morphèmes, d'attribuer des appartenances de ces morphèmes à une langue plutôt qu'à une autre : tout se passe comme si les problèmes théoriques d'identification d'une langue matrice et d'une langue insérée, de catégorisation de formes hybrides, de différenciation des « codes » dans le code-switching ou au contraire de «mélange » des « codes » dans le code-mixing étaient résolus dans la transcription, avant même d'être soumis à l'analyse des données. Ces phénomènes montrent l'intérêt de réfléchir aux formes de codification imbriquées dans les conventions de transcription et, plus radicalement, indiquent que ces dernières sont l'émanation et la matérialisation de la théorie du CS adoptée par le transcripteur.

Un autre enjeu de la transcription est celui de la pertinence de son détail - amplement démontrée par les travaux de l'analyse conversationnelle en linguistique, notamment en ce qui concerne la manière dont le formatage des tours est accompli par les locuteurs pas à pas, de manière incrémentale et temporellement précise. Voici un exemple de la manière dont la précision de la transcription peut affecter radicalement l'analyse ${ }^{7}$, tiré d'une analyse de Steensig $(2003,809)$ d'une interaction entre jeunes turcs au Danemark :

(1) (Steensig, 2003)

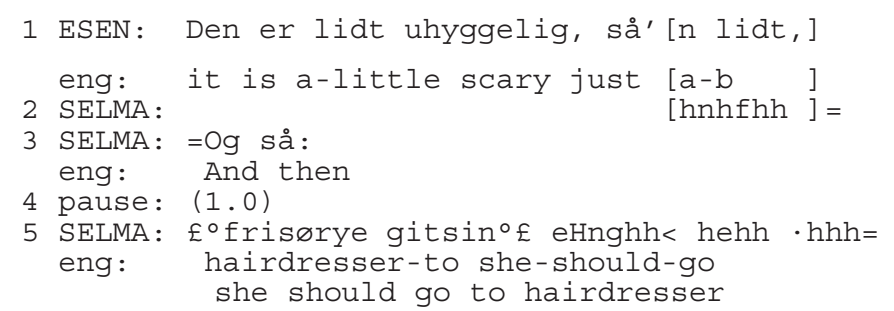

La première transcription avait noté les lignes 3-5 de la manière suivante :

(2) (cf. extrait 1 , lignes 3-5)

SELMA : og så frisørya gitsin

Sur la base de cette transcription, on pourrait traiter ce que dit Selma comme un énoncé où les deux langues, le danois et le turc, sont parfaitement imbriquées, rendant difficile l'identification d'une langue de base et d'une langue insérée.

La seconde transcription, fournie par Steensig, montre autre chose : après un début de tour en danois (3) qui projette une suite, Selma effectue une longue pause (4), suite à laquelle elle reprend la parole : ligne 5, elle parle avec une voix différente - souriante et plus basse - qui contraste avec la ligne 3. Elle utilise un mot danois, "frisør » qu'elle insère dans un cadre syntaxique en turc (le suffixe est turc ainsi que le verbe). La deuxième transcription permet donc une analyse très différente : un premier fragment est proposé en danois, puis un second, après la pause, opère un changement de langue, en adoptant le turc comme langue de base. La raison de ce changement peut être esquissée en observant le contexte séquentiel de ce tour: Selma a déjà essayé d'introduire un nouveau topic précédemment, sans succès ; c'est ici sa deuxième tentative, qui a lieu alors que Esen est encore en train de traiter un autre topic avec un autre co-participant. L'alternance du danois au turc intervient ici avec d'autres ressources (la pause, la projection d'une suite) comme un moyen supplémentaire pour regagner le floor et l'attention des co-participants.

De manière intéressante, Torras \& Garafanga (2002) adoptent dans leurs conventions un symbole typographique supplémentaire pour les lexèmes dont l'appartenance à une langue n'est pas décidable.

${ }^{7}$ Voir Wei (2002 : 174-175) pour un exemple de reprise d'une ancienne transcription revue et corrigée selon les standards de l'analyse conversationnelle et donnant lieu à une révision de ses résultats. 
On voit bien avec cet exemple que les détails de la transcription interviennent à la fois pour rendre compte de la segmentation du flux de la parole dans le temps, pour catégoriser les segments ainsi délimités et pour fournir une interprétation fonctionnelle qui tienne compte du positionnement de cette parole par rapport à l'organisation séquentielle et aux actions en train de se faire.

\subsection{Identité, appartenances catégorielles, contexte}

Le CS est aussi un terrain idéal pour questionner les approches de l'identité et du contexte dans l'analyse des pratiques langagières - puisqu'une des fonctions classiquement attribuée au CS est d'indexer une appartenance à un groupe ou à une culture. Cette affirmation apparemment évidente est en fait susceptible d'interprétations très diverses, selon que l'on épouse plutôt une vision essentialiste de l'identité - pour laquelle l'individu porte avec soi son identité propre, qui en retour structure voire détermine ses actions - ou une vision praxéologique et située de l'identité - pour laquelle l'appartenance à une catégorie ou à une autre est élaborée, revendiquée, exhibée, déniée, déplacée de manière localement située au sein de cours d'actions sociales. La première conception permet de fonder une vision stabilisée de l'identité, la seconde de rendre compte de la variabilité et de la mobilité des identités. La première privilégie souvent des méthodologies d'enquête recourant à des sources d'information extérieures à l'activité ou à l'interaction étudiée (p.ex. des entretiens avec les locuteurs, des récits de vie, etc.), la seconde privilégie l'analyse de la structuration de l'interaction, en tant que lieu où l'identité est élaborée de manière pertinente par rapport aux contingences et aux finalités locales de la parole et de l'action en train de se faire.

Le passage de l'une à l'autre opère un renversement de perspective: au lieu d'utiliser les connaissances ethnographiques concernant l'identité de l'un ou de l'autre participant pour expliquer la manière dont ils opèrent leur CS et indexer le second sur le premier, il s'agit d'observer la façon méthodique dont ils exploitent le CS parmi d'autres ressources pour la structuration de leurs tours et de leurs séquences afin de reconstruire les positionnements qu'ils construisent et exhibent vis-à-vis de ces ressources, traitées par eux - de manière locale et contingente. En retour, cela interroge critiquement la place des connaissances ethnographiques comme ressources explicatives de l'analyste (ces ressources ethnographiques étant elles-mêmes fabriquées dans des pratiques de recueil de la parole des informateurs, p.ex. dans des entretiens où les questions de l'intervieweur jouent un rôle structurant dans l'émergence des catégories pertinentes, voire dans des expériences participatives où l'enquêteur s'engage plus ou moins dans un processus d'apprentissage et de transformation en tant que «nouveau membre »).

Les revendications identitaires manifestées dans et par le CS ont souvent été traitées, surtout depuis son usage par Gumperz (1982), dans les termes de la dichotomie entre "we code » versus «they code ». Or, on constate des usages très divers de cette opposition dans la littérature. D'une part, elle est invoquée pour indexer les valeurs identitaires de deux codes employés dans le CS - le «we » correspondant souvent à une position minoritaire dominée et le «they» à une position majoritaire dominante: elle est alors une ressource analytique efficace, puisqu'elle permet d'associer les langues en présence (souvent deux, dans les analyses présentées dans la littérature, même si le CS peut comporter bien davantage de variétés, voir Finlayson \& Slabbert 1997) avec une bipartition des groupes qui homogénéise la communauté d'appartenance du locuteur aussi bien que les autres communautés. Or, la notion de "communauté langagière »et son homogénéité ont été critiquées depuis longtemps (voir Irvine, 1987), ainsi que la structuration des groupes en entités fixes et délimitées: les identités individuelles et la définition des groupes sont des accomplissements dynamiques, pouvant être reproduits ou transformés au gré des activités sociales et des positionnements émergeant de manière contingente et locale dans l'interaction. De ce point de vue, c'est donc un usage alternatif du " we code » $v s$ « they code », très différente de la première, qui peut être esquissé (Sebba \& Wootton 1998) : la distinction binaire est une ressource qui peut être mobilisée de manière $a d$ hoc dans l'interaction, mais qui est alors configurée localement dans la 
parole des participants, assignant à toutes fins pratiques des positions, ainsi que des affiliations et désaffiliations, à des présents et à des absents. "We » et «they » ne sont alors à aucun moment des identités ou des références fixes associées de manière stable à des « codes » qui les indexeraient ou les actualiseraient. Ce sont plutôt une manière parmi d'autres de produire localement du sens dans leur mobilisation et agencement spécifique à la séquence en cours.

Si la ressource «we » $v s$ «they » peut permettre de produire des contrastes et des oppositions aux fins pratiques de l'activité interactionnelle en cours, les traiter comme des ressources signifie précisément ne pas considérer que les situations bilingues sont structurées a priori de manière binaire, p.ex. par le conflit entre communauté d'origine et société d'accueil, entre langue maternelle et langue cible ${ }^{8}$. Ces oppositions sont battues en brèche ${ }^{9}$ par des usages du CS dans lesquels les locuteurs mobilisent tour à tour les deux codes comme « we code » (comme les Jamaïcains utilisant le London English et le London Jamaican étudiés par Sebba \& Wootton 1998) ou bien dans lesquels les locuteurs utilisent la langue des communautés migrantes auxquelles ils n'appartiennent pas: c'est le cas des usages du créole chez les Anglais blancs du sud de Londres décrit par Hewitt (1986), ou des usages du turc par des jeunes Allemands décrit par Auer \& Dirim (2000). Ces données invitent à prendre en considération la manière dont les langues disponibles sont localement mobilisées pour accomplir/négocier/réélaborer des positionnements interactifs, énonciatifs, identitaires, sociaux, etc. dans l'interaction. La mobilisation des langues comme autant de ressources prenant leur sens de manière localement située est particulièrement visible dans les phénomènes de «crossing» étudiés par Rampton (1995). Dans ses données, des adolescents jamaicains de Londres recourent ponctuellement à des éléments de penjabi, alors que leurs interlocuteurs indiens mobilisent des éléments de créole : cette exploitation des ressources de l'autre suspendent les appartenances dans des moments sociaux et interactionnels «liminaires》- un concept emprunté à Turner (1982) et à son analyse des rites d'initiation. Cette liminalité caractérise des moments interactionnels de transition, de relâchement des normes interactionnelles, de blague, de jeu, de drague, caractérisés à la fois par une relative indétermination des rôles de chacun et par une forte polyphonie des paroles échangées. Dans ces moments ont lieu les phénomènes de «crossing », qui s'orientent donc vers cette ambiguïté ponctuelle pour explorer les ressources culturelles et linguistiques des « autres ». Cela ne signifie pas nécessairement une subversion ou un renversement durable des différences ethniques, mais marque des contextes qui échappent ponctuellement à des frontières. L'intérêt de ces cas, tout comme les cas de "passing 》 (croisant souvent d'ailleurs gender et ethnicité, cf. Garfinkel 1967:136-7; Hewitt 1986:165, 195 ; voir en particulier la notion de « ethnic passing » chez Bucholz 1995) consiste à montrer la flexibilité des ressources plurilingues qui ne peuvent être a priori assignées à des marquages symboliques stables.

C'est ainsi que les recherches sur le parler bilingue ont contribué à repenser l'articulation entre langage et identité. A travers des choix linguistiques (choix de lexique, de grammaire, de prononciation...), les locuteurs proclament, revendiquent, ou changent, leur identité, en s'associant ou en se distanciant de groupes de référence, identifiés à travers des matériaux linguistiques particuliers ${ }^{10}$.

\footnotetext{
${ }^{8}$ Dans ce sens, comme le montre Goodwin (1999), le conflit n'est pas inhérent à des groupes ou à des catégories identitaires, ethniques ou de gender, mais est construit par des ressources situées (Goodwin 2003).

${ }^{9}$ Voir aussi les cas de réversibilité forte entre une langue et l'autre dans le CS, montrant qu'elles sont interchangeables pour l'accomplir de contrastes fonctionnels dans l'interaction, sans pour autant indexer des valeurs pré-déterminées - donc en reconfigurant les contextes interactionnels qu'elles servent à décrireorganiser (Alfonzetti 1998: 186-207).

${ }^{10}$ "Linguistic behaviour as a set of acts of identity in which people reveals both their personal identity and their search for social roles » (Le Page \& Tabouret-Keller 1985: 14).
} 
Dans une perspective ethnométhodologique et conversationnaliste, on soulignera la manière dont l'identité est accomplie pas à pas dans la parole et l'action en interaction. Cela signifie que d'une part l'identité est élaborée par les participants au sein de l'organisation séquentielle de la parole, en rendant certains détails pertinents et conséquentiels pour la production de la suite de l'interaction (Schegloff 1992). Méthodologiquement, cela prévient l'imposition par l'analyste de catégories exogènes à l'interaction et l'invite plutôt à repérer les pertinences rendues localement et publiquement manifestes par les participants dans ce qu'ils font (et pas nécessairement dans ce qu'ils en disent qu'ils font) (Antaki \& Widdicombe 1998). D'autre part, cela invite à observer les orientations des autres participants vers ces tissus de pertinences, ainsi que leur acceptation/refus, affiliation/desaffiliation, alignement/désalignement par rapport aux positionnements implémentés dans des tours auxquels les co-participants offrent des réponses et des enchaînements. Ainsi Day (1994) parle de processus d'ethnification - ascribing identity predicates - accomplis tour par tour et par rapport auxquels l'interlocuteur peut résister, diverger, refuser. De même, en réparant l'usage d'une forme linguistique ou le recours à une langue plutôt qu'à une autre, un locuteur peut évaluer l'(in)compétence de son partenaire et par là le re-catégoriser en termes non convergents avec le choix de langue que celui-ci avait effectué (Cashman 2002, 2005 ; Mondada 2000b).

Par ailleurs, il est aussi important de considérer que le fait de choisir une langue plutôt qu'une autre ne signifie pas toujours revendiquer une affiliation ethnique ou la rejeter, mais peut accomplir un travail interactif autre que la catégorisation. L'affiliation à un groupe ou à une identité dans le CS ne doit donc pas être postulée mais démontrée sur la base de l'orientation des participants telle que déployée dans ce qu'ils font effectivement. La revendication d'une affiliation catégorielle est quelque chose qui se fait dans les actes et non quelque chose qui est, émanant d'une relation spéculaire pré-déterminée entre formes linguistiques et appartenances socio-ethniques.

Dans ce cadre, le recours à l'analyse des dispositifs de catégorisation (Membership Categorization Analysis) initiée par Sacks (1972) est un complément indispensable à l'analyse séquentielle : son point de départ est en effet le double constat que toute personne est susceptible d'un nombre élevé de descriptions possibles (selon les collections du genre, de la profession, de la religion, de l'ethnie, de la religion, etc.) et qu'une description catégorielle référentiellement correcte n'est pas nécessairement pertinente pour l'activité en cours. Le problème soulevé par Sacks est donc de savoir comment une catégorie est rendue pertinente par les participants dans l'interaction à travers des procédés de catégorisation qui ne consistent pas uniquement à nommer explicitement une catégorie mais aussi à "doing being » une certaine catégorie, voire à faire fonctionner des inférences sur la base p.ex. de la référence à des «category-bound activities » (Sacks 1972, 1992 ; Hester \& Eglin 1997 ; voir à propos des interactions plurilingues Mondada 1999, 2004 ; Garafanga 2001, 2005).

Là encore, la transcription est un lieu où la perspective de l'analyste se matérialise dans le dispositif de représentation des données: la transcription est en effet souvent précédée de quelques lignes de description ethnographique, « contextualisant » l'extrait : ces lignes peuvent effectuer une projection, sur les données à venir, d'une interprétation contextuelle exogène propre à l'enquêteur qui éclairera le texte de la transcription d'une manière particulière. En outre, dans la transcription elle-même, la manière dont les participants sont traités est significative (Mondada 2002) : plusieurs options existent quant à la manière d'identifier les participants au début de chaque prise de parole, pouvant privilégier leurs noms, leurs catégories socio-professionnelles ou encore leur langue d'origine..., i.e. pouvant être plus ou moins indéterminées quant à certaines dimensions, traitées ainsi par les choix de transcription comme étant données a priori ou comme devant être reconstituées sur la base de la transcription elle-même.

Si l'on se penche sur les transcriptions d'un article de Myers-Scotton (1988) montrant que le CS sert à indexer l'appartenance identitaire, souvent ethnique, des participants, on remarque que les locuteurs y sont variablement identifiés, selon leur profession (dans la plupart des exemples), selon 
leur sexe ("He/She") dans un échange où un homme invite une femme à danser (1988, note 11), ou selon leur langue d'origine. La mention de la langue ou des langues parlées dans le tour figure entre parenthèses et suit la catégorie ethnique du locuteur:

\section{(3) (Myers-Scotton, $1988: 176)$}

Setting: Four young Kenyan men who have completed secondary school and who work in the same government ministry in Nairobi are chatting. Two are native speakers of Kikuyu, one of Kisii, and one of a Kalenjin language. Swahili and/or Swahili/English are unmarked choices.

Kikuyu I (Swahili): Sasa mumesema nini juu ya hiyo plan yetu? Naona kama siku kama siku zinaendelea kwisha. ("Now, what do you all say about the plan of ours? I think time is getting short")

Kikuyu II (Swahili; English): Mlisema tu collect money, lakini hakuna mtu hata mmoja ambaye amenipatia pesa. ("You said collect money, but there isn't even one person who has got money for me")

Kalenjiin (Swahili): Makosa ni jako kama mweka hazina. [...]. ("This fault is yours as treasurer. [...])

La question de la pertinence est posée dès la courte description du contexte précédant la transcription, qui mentionne plusieurs catégories (l'âge, la formation, la profession, la langue, la relation d'amitié,...). L'exemple veut montrer que le CS peut avoir un effet d'exclusion des personnes qui ne parlent pas la langue (les locuteurs Kisii et Kalenjin vont se plaindre plus loin du fait que les locuteurs Kikuyus switchent dans leur langue). Mais l'extrait commence par une discussion où les catégories pertinentes pourraient relever de l'appartenance professionnelle plutôt que de l'appartenance ethnique ou linguistique (les deux tendant à être confondues dans la notation des identités). De même, la catégorisation de l'activité dans le texte introductif - "chatting" - ne semble pas coïncider avec celle des interactants, qui s'orientent plutôt vers l'activité "résolution de problème". Si le CS est lié, comme Myers-Scotton le dit elle-même, à des identités multiples (1988: 176), il est difficile de traiter cette multiplicité en privilégiant une identité unique et constante dans la transcription.

Les questions de description pertienente du contexte et des identités se glissent donc à tous les niveaux de l'analyse, dès l'enregistrement, la préparation et la transcription des données. Ces questions interviennt de manière doublement fondamentale dans l'interprétation des phénomènes de CS : d'une part ces phénomènes sont sensibles au contexte, qu'ils contribuent réflexivement à configurer; d'autre part, la conception du contexte et de l'identité qui fonde l'analyse est susceptible de configurer à son tour l'interprétation qui sera donnée à ces phénomènes. Cela invite à une problématisation des données et des cadres interprétatifs qui en rendent compte.

\section{Le CS comme ressource pour l'organisation endogène de l'ordre interactionnel}

Dans ce qui suit, nous allons analyser une série d'extraits d'interactions professionnelles en contexte international où nous reformulerons empiriquement les enjeux concernant la définition locale du contexte, l'orientation vers les ressources plurilingues disponibles et leur exploitation et interprétation indexicales au fil de la séquentialité de l'interaction. Le corpus est constitué de séances de concertation entre chirurgiens français (Strasbourg, Toulouse), belges (Bruxelles), suisses (Bâle) et allemands (Freiburg) qui se rencontrent hebdomadairement par visioconférence avec l'objectif de discuter de cas cliniques difficiles et d'améliorer, par la discussion, les modes de prise de décision en ce qui concerne les procédures, les techniques et les traitements chirurgicaux.

Nous nous intéresserons ici à la manière dont s'opère le CS dans ce contexte, en nous penchant sur l'exploitation de ressources plurilingues dans des moments séquentiels centraux de l'activité étudiée : l'ouverture (5.1), la présentation de l'ordre du jour (5.2), la discussion (5.3) et la gestion de problèmes techniques soulevés par le dispositif de la visioconférence (5.4). Nous montrerons que 
s'il est difficile d'attribuer une fonctionnalité pragmatique unique ou spécifique à une langue ou aux usages d'un locuteur, ainsi que de déterminer une association stable entre un type d'activité et une langue, voire un ensemble d'attentes et de droits et obligations caractéristiques d'un type d'activité, il est en revanche possible de rendre compte des choix de langue des locuteurs au fil du déroulement séquentiel de l'interaction.

\subsection{Ouvertures et choix de langue}

L'ouverture est un moment crucial pour l'organisation de l'interaction : c'est le moment où les participants organisent leur entrée conjointe dans l'échange, où ils accomplissent l'alignement de leurs identités, de leur définition du contexte et de leurs conduites en vue de l'activité collective à venir (Schegloff 1972, Zimmerman 1992).

L'ouverture est aussi le lieu où le choix de(s) langue(s) de l'échange est accompli par les participants : cela participe de l'accomplissement de leur alignement mutuel. Analyser l'ouverture d'une interaction permet ainsi de traiter l'adoption ou la négociation de une ou plusieurs langues de l'interaction comme un accomplissement localement situé des participants - et non par exemple comme quelque chose de décidé à l'avance de manière réglementaire ou comme donné par les identités des participants voire par le contexte de l'échange.

L'établissement du contact visiophonique est ici du ressort technique de Strasbourg, où Séford (SEF) officie en tant que chairman de la réunion. Dans l'extrait suivant, Séford vérifie la connexion avec Freiburg en Allemagne, où se trouve son collègue Oberwieser (OBE) :

(4) TC20117)

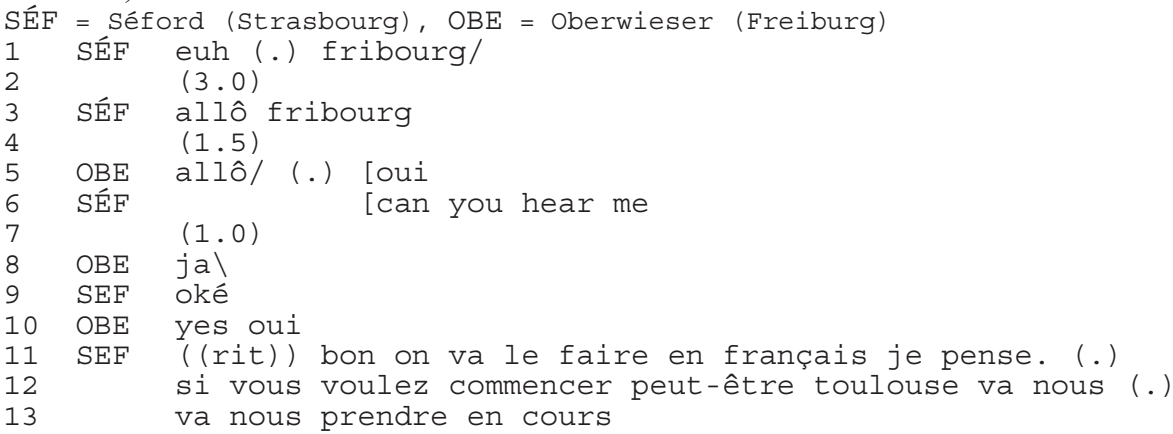

Dans cet extrait, Séford s'adresse interrogativement au site de Freiburg en français : son premier summons (1) ne reçoit pas de réponse (2) et est renouvelé, toujours en français, après une pause (3). Après une nouvelle pause, il reçoit finalement une réponse (5), en français aussi. Cependant, cette réponse est chevauchée par une nouvelle tentative de Séford, orientée vers la pause qui la précède, traitée comme une absence de réponse (4) : cette fois, Séford passe à l'anglais pour produire son troisième summons (6). Bien que Oberwieser ait déjà répondu (5), cette réponse n'est pas entendue ou prise en compte par son interlocuteur: Oberwieser s'aligne avec le changement de langue de Séford, en proposant à nouveau une réponse, qui elle aussi change de langue, mais choisit l'allemand plutôt que l'anglais (8). Alors que Séford ratifie la réponse en français (9), Oberwieser offre un prolongement de sa réponse, cette fois en anglais et en français (10), ce qui suscite le rire de Séford.

En réalisant de manière étendue une séquence qui pourrait être réalisée en deux tours, cette expansion de la séquence initiale summons/answer s'oriente de manière manifeste vers les choix de langue envisageables dans ce contexte : la reprise du tour de ratification exhibe un parcours de tous les choix linguistiques possibles. De cette manière, Oberwieser accomplit au moins deux choses : 
d'une part il exhibe une ouverture maximale des ressources linguistiques disponibles pour l'échange, en laissant le soin à son interlocuteur de choisir la ressource dans laquelle il enchaînera au tour suivant (ce que fait Séford à la ligne 11, à la fois en parlant français et en thématisant le choix de langue) ${ }^{11}$. D'autre part, ce faisant, il s'exhibe en tant que locuteur plurilingue en action (son identité est accomplie par le procédé consistant à 'doing being plurilingual') (Mondada 2004).

Cette «mise à plat» des ressources linguistiques disponibles par Oberwieser, dans des tours exhibés comme redondants par rapport à la résolution du problème interactif, repose sur la reconnaissabilité aproblématique des différentes manières de dire « oui »/ « ja »/ « yes » et donc sur une catégorisation tacite de ces formes comme appartenant à des langues différentes. Il n'y a ici aucune hybridation des ressources linguistiques: au contraire les participants s'orientent vers une pluralité de langues traitées comme clairement distinctes et vers le choix exclusif de l'une ou de l'autre. Ces propriétés sont visibles dans le traitement successif de l'ordre du jour, que nous allons analyser ci-dessous : même si la langue dans laquelle se feront les interventions successives est renégociée plusieurs fois, sa catégorisation et son identification sont traitées comme évidentes (cf. infra), et les participants s'orientent vers un modèle normatif endogène d'usage d'une langue à la fois, le choix de l'une excluant l'autre ${ }^{12}$.

\subsection{Annonce et planification des activités et enjeux participatifs des choix de langue}

Les activités professionnelles comme les réunions sont souvent considérées comme étant structurées par des contraintes externes, comme des convocations, des ordres du jour, voire des actions planifiées attribuées à des rôles hiérarchiques. L'analyse des procédés par lesquels la réunion se structure pas à pas dans l'interaction montre que, malgré ces dispositifs organisationnels, la réalisation des points de l'ordre du jour est un accomplissement situé moment par moment au fil du déroulement de la réunion.

Cela concerne aussi la gestion des choix de langue : dans le cas empirique analysé ici, les réunions sont annoncées par un ordre du jour qui prévoit non seulement l'ordre et le contenu d'une série d'interventions mais aussi la langue de l'échange. Dans la réunion dont est extrait le fragment suivant, l'ordre du jour envoyé préalablement aux participants prévoyait l'anglais comme langue de la réunion. Nous verrons que cela n'exclut pas du tout une renégociation des langues à utiliser dans les différentes interventions :

(5) (TC07058)

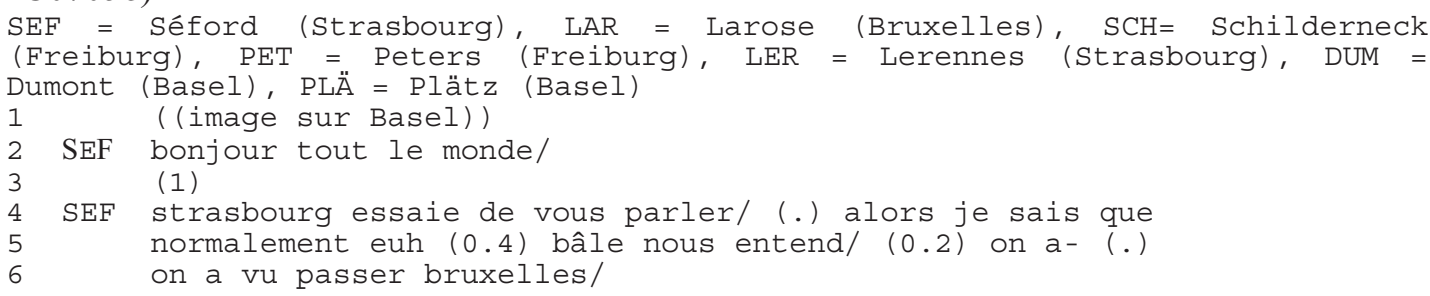

${ }^{11}$ Heller (1982) cite de nombreux exemples tirés d'ouvertures d'appels téléphoniques dans des entreprises et institutions à Montréal où l'appelé formate systématiquement son tour initial de sorte à laisser le choix de la langue à son interlocuteur (français ou anglais). Le formatage du tour qui exhibe les orientations particulières des participants vers un type de choix.

12 Dans ce sens, les participants s'orientent normativement vers des activités réalisées de manière monolingues et non vers des activités qui pourraient être caractérisées par du code-switching. Cela est ultérieurement démontré par les nombreuses auto-réparations de la langue en début de tour, comme dans l'exemple suivant :

(12028) (DUM répond à une question)

DUM non non (0.5) C'est les inter-(.) c'est les internistes qui ont gard- euh s- sorry the colleague of the intern meditsine euh kept the NEW scan 


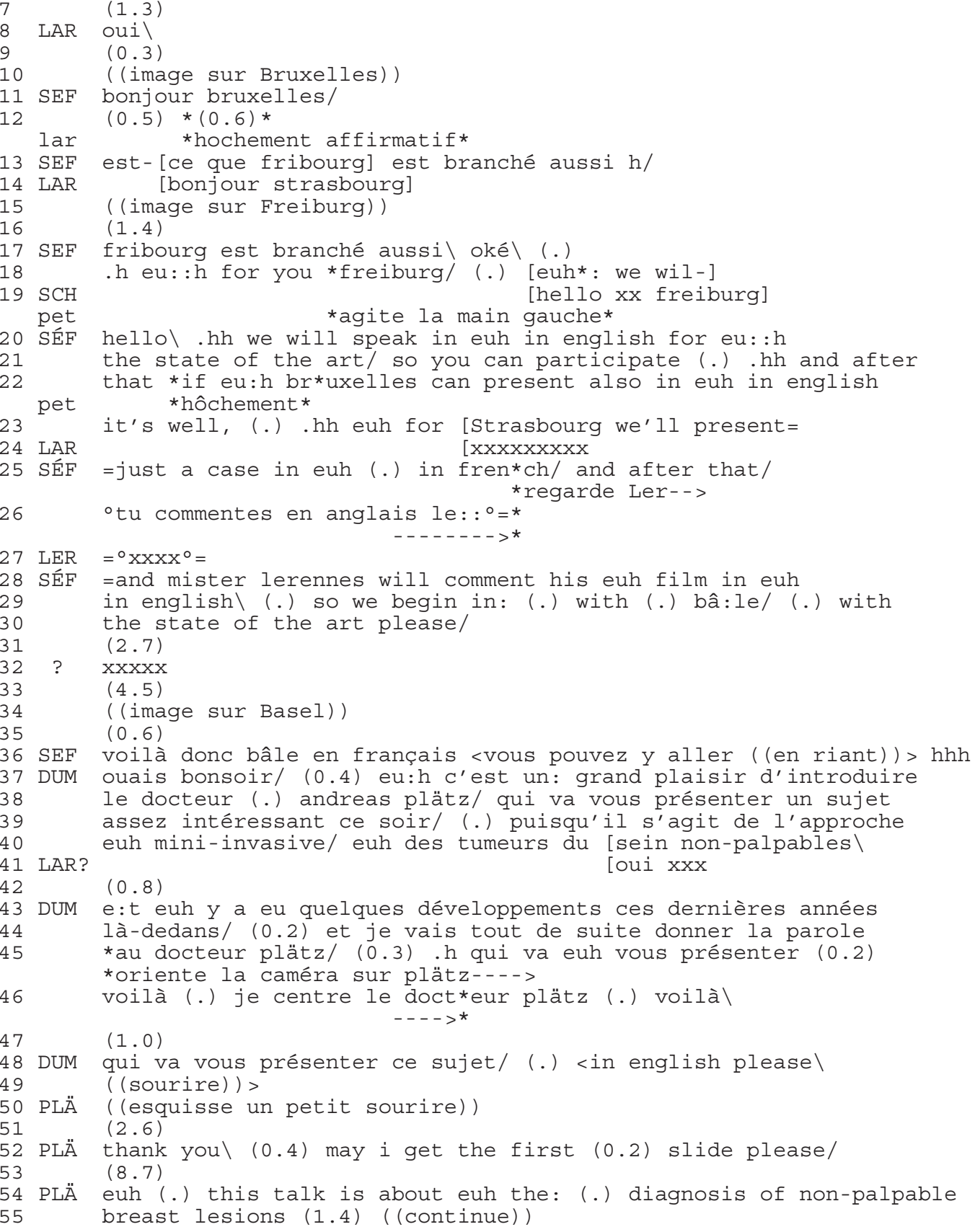

Cet extrait documente la transition entre l'ouverture et la planification de la réunion. Plusieurs enjeux apparaissent, permettant de montrer d'une part comment les caractéristiques plurilingues de l'événement sont à la fois exhibées et thématisées, et d'autre part comment les choix de langue sont négociés d'une manière qui est participant-related, pour reprendre la terminologie de Auer, en s'orientant vers les conditions de participation d'un groupe d'interlocuteurs particuliers, les médecins allemands de Freiburg, et vers la compétence qui leur est attribuée (censés ne pas comprendre le français).

L'extrait commence avec un échange de salutations, configuré spécifiquement pour ce type d'échange médié technologiquement: les salutations y deviennent une paire adjacente qui fonctionne comme une ressource pour vérifier que la connexion visiophonique est effectivement 
établie. C'est ainsi que la salutation adressée à tous les participants (2), ne recevant pas de salutation en retour (3), est transformée en une vérification explicite du contact (4). Dans le cas de Bruxelles, une paire adjacente est consacrée à la vérification-confirmation du contact (6-8), rendant ainsi possible un échange de salutations, adressées au site (nom de la ville) plutôt qu'à ses occupants (11, 14).

Dans le cas de Freiburg, cette vérification est implémentée de manière quelque peu différente : la question de Séford (13) reçoit un type particulier de réponse, fourni par l'apparition de l'image de Freiburg à l'écran (15), thématisé dans le tour suivant par Séford (17). Dans cette séquence, Séford réfère à Freiburg en français et à la troisième personne, sans s'adresser au site et sans le saluer après vérification du contact. En revanche, il s'adresse à lui en changeant de langue («for you freiburg » 18) : le CS se présente ici clairement comme participant-related, en allant de pair avec le passage de la troisième à la deuxième personne. Ce changement est traité par Schilderneck comme une opportunité de placer une salutation (19) à laquelle répond son interlocuteur, par une deuxième partie de paire insérée dans son tour (20). Séford traite l'anglais non seulement comme une ressource pour s'ajuster au destinataire allemand, mais plus généralement aussi comme matérialisant une orientation qui configure le formatage de la suite de son long tour, voire de la réunion tout entière : il présente en effet le programme de la réunion en associant systématiquement chaque point de l'ordre du jour à un choix de langue orienté vers la participation des Allemands. De cette manière, Séford accomplit pratiquement la catégorisation des Allemands comme "participants non francophones ${ }^{13}$ (que Peters accepte par son hochement positif de la tête positionné juste après la mention de la participation).

De manière intéressante, la sélection de l'anglais comme langue de la réunion n'est pas effectuée une fois pour toutes et pour l'événement dans son ensemble. Au contraire, le choix de l'anglais est vérifié et négocié pour chaque présentation avec les intervenants concernés, de manière spécifique à chacun. Ainsi, le choix de l'anglais est présenté par Séford comme aproblématique pour le state of the art (20-21), mais de manière hypothétique pour la présentation de Bruxelles (22-23) tout en n'attendant pas l'accord de Larose (24) pour continuer ; la présentation de Strasbourg (le site auquel appartient Séford) est annoncée en français, avec une légère hésitation (25), alors que la langue de la dernière présentation est négociée dans une séquence insérée avec Lerennes, qui est assis à côté de Séford à Strasbourg (26-27), avant d'être annoncée officiellement (28-29). Ce fragment montre que même si l'ordre du jour est présenté comme ayant été programmé auparavant, la langue des différentes interventions est vérifiée cas par cas de manière locale et localement renégociable. Même si l'anglais est présenté comme la solution qui permet aux Allemands de participer, le choix de l'anglais comme langue de la réunion doit constamment être répété et ré-accompli, confronté à des alternatives possibles, définissant la réunion davantage comme un événement plurilingue que comme un événement en anglais lingua franca.

L'annonce de la première intervention, par Bâle, montre que la langue de l'événement - tout en ayant été annoncée auparavant comme devant se passer en anglais (20-21) - fait à nouveau l'objet d'une négociation locale au moment de sa réalisation. En effet, la présentation de Séford montre que l'anglais ne va pas de soi :

(6) (= extrait 5, lignes 29-30) so we begin in : (.) with (.) Bâle/ (.) with the state of the art pleasel

\footnotetext{
${ }^{13}$ Bien que dans de rares occurrences enregistrées dans d'autres réunions la catégorie de «participants non germanophones » puisse aussi être rendue pertinente, la catégorie «participants non francophones » est nettement plus fréquente - montrant ainsi qu'un type de catégorisation n'entraîne pas automatiquement ni symétriquement d'autres types de catégorisation pouvant en être inférés.
} 
Alors que la mention de la langue est projetée en premier par la préposition « in: », celle-ci n'est pas suivie par son argument, mais par une autre préposition, " with », qui introduit d'abord le site et puis le type de présentation. En outre, on remarquera que le nom de la ville est introduit en français (alors qu'il pourrait l'être en allemand, voire en anglais): Séford s'oriente ici vers la langue préférée du responsable de Bâle, qui est le français. Cette association de Bâle et de la langue française est d'ailleurs rendue explicite par Séford alors que la prise de parole de ce site est retardée (« voilà donc bâle en français <vous pouvez y aller ((en riant))> hhh» 36). On remarquera le rire qui accompagne cette mention, s'orientant vers le caractère problématique du choix de langue.

Le représentant de Bâle prend effectivement la parole en français (37) sans thématiser ce choix de langue, largement introduit par ce qui précède. Dumont toutefois n'est pas l'auteur de l'intervention annoncée, mais uniquement son représentant local, introduisant à son tour le docteur Plätz : celui-ci est présenté par le sujet de son exposé (38-44), puis par une centration de la caméra sur lui (45-46) et enfin, en tout dernier lieu, par la langue qu'il utilisera ( «<in english please $\backslash(($ sourire $))>\gg$ 48). Plätz ne thématise pas la langue, mais esquisse un sourire (49-50), avant de prendre la parole et de commencer son exposé en anglais.

Dans cet extrait, on voit donc que le choix de langue, tout en étant annoncé, planifié, programmé, fait l'objet de choix localement situés, et est un accomplissement local et contingent au fil du déroulement de l'interaction : même si la réunion a été programmée en anglais, sa réalisation montre que l'anglais ne va pas de soi ; même si une présentation peut être annoncée comme étant en anglais au début de la réunion, le choix de langue peut être accompli et négocié à nouveau lors du début de la présentation elle-même ${ }^{14}$. Dans ce sens, le choix de langue reste un accomplissement indexical et contingent, effectué au fil de l'organisation séquentielle, tour par tour, unité de construction de tour par unité de construction du tour.

En outre, le fait que la mention de la langue choisie soit, dans le site de Bâle, modalisée par des rires $(36,48)$ manifeste un rapport particulier à ce choix ainsi qu'à la langue elle-même. Dumont mentionne l'anglais avec un fort accent français à la ligne 48, outre qu'avec un sourire: si les participants catégorisent ce choix de manière très explicite et indiscutable comme concernant de l' «anglais» (sans aucune modification ou modalisation de sa dénomination), ils incarnent aussi, dans la prononciation et dans les mimiques faciales, une orientation distancée vers un anglais langue seconde, anglais international bricolé à toutes fins pratiques, anglais comme langue étrangère.

\subsection{Choix de langue dans la discussion et modes de participation}

Bien que l'anglais soit introduit explicitement à plusieurs reprises comme la langue de la réunion, les choix de langue continuent à se faire de manière localement et séquentiellement située au fil de la réunion. Afin de mieux cerner la diversité de ces choix et surtout leur organisation séquentielle, nous allons nous pencher sur la discussion qui suit le cas présenté par le docteur Plätz, sur lequel se terminait l'extrait précédent.

\footnotetext{
${ }^{14}$ Voir par exemple le début de la présentation de Bruxelles, annoncée comme étant hypothétiquement en anglais dans l'extrait que nous avons analysé :

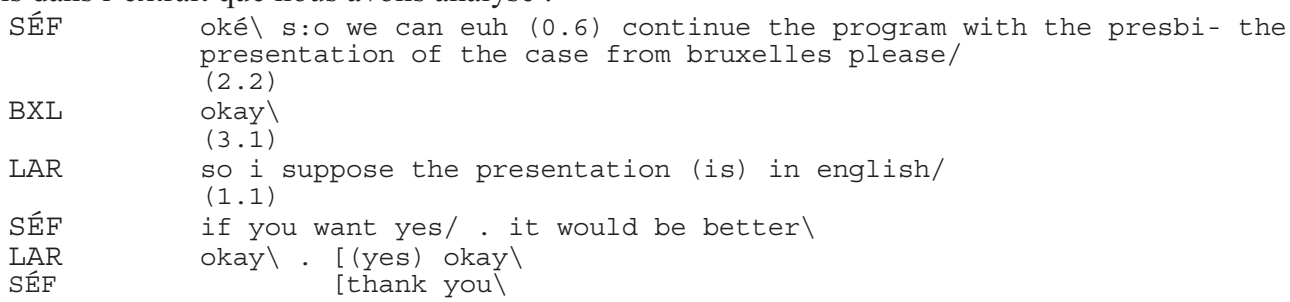


(7) $(\mathrm{TC} 07058 / 9.50)$

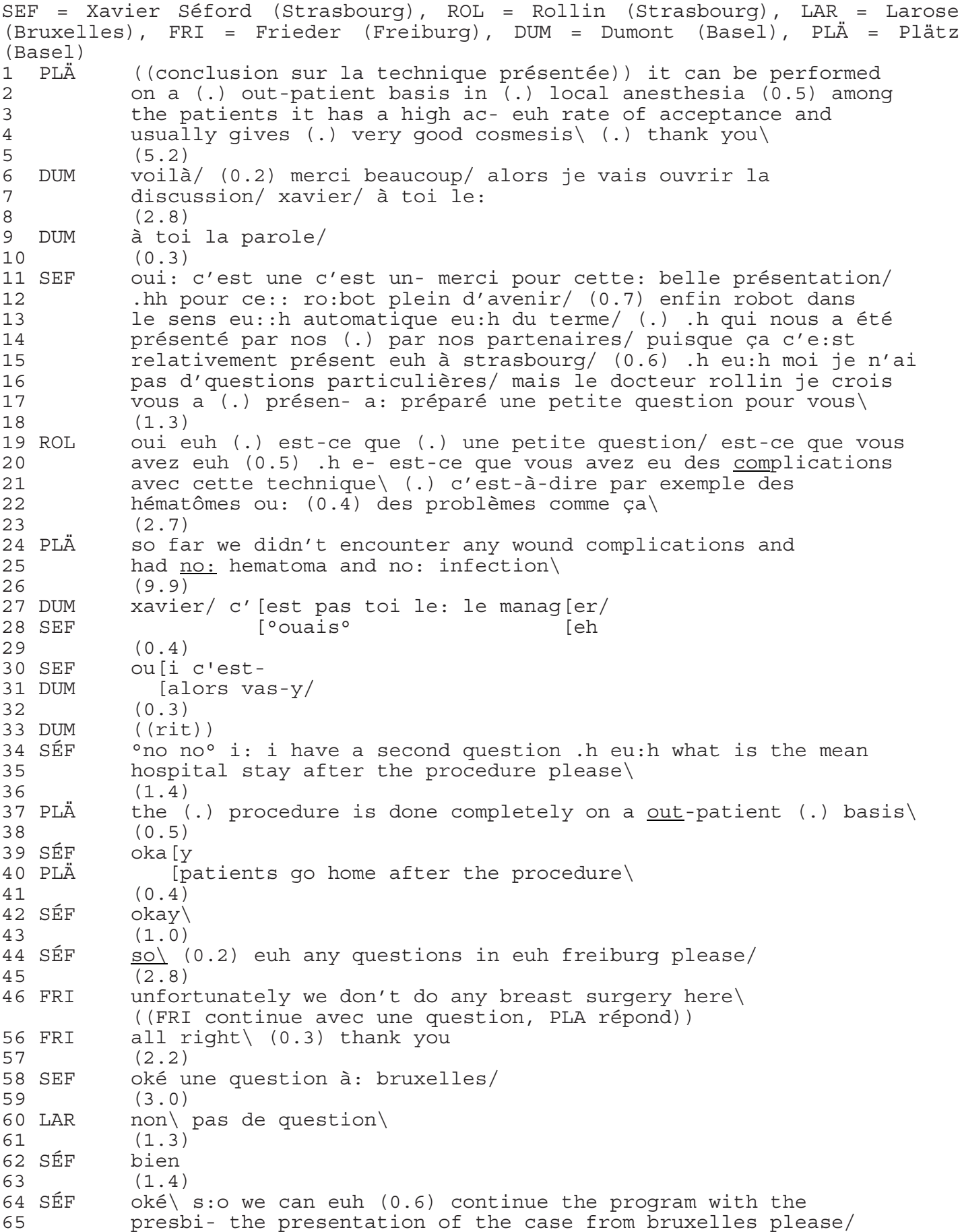

La fin de l'exposé de Plätz est symétriquement suivie par une intervention de Dumont qui tout en effectuant la clôture de l'exposé et l'ouverture de la discussion donne la parole à Séford qui est le modérateur de la séance. On remarquera au passage que ce type d'organisation redéfinit les activités liées à la catégorie du modérateur ainsi que la personne qui occupe cette catégorie, provoquant une série de problèmes dans la gestion des tours de parole (6-11, voir aussi plus bas, 26-33). Dumont accomplit donc le passage de l'exposé à la discussion en français, et Séford s'aligne avec ce choix 
en offrant une évaluation de la présentation et en donnant la parole au premier intervenant dans la discussion, Rollin, projetant sa prise de tour en français. Après la question que celui-ci pose en français, Plätz répond en anglais (24-25). Ce dernier exhibe ainsi, dans cette réponse aussi bien que dans les suivantes, un maintien de l'anglais en seconde partie de paire adjacente indépendamment de la langue dans laquelle a été formulée la première partie.

Cette première paire Question-Réponse est suivie d'un nouveau problème de gestion de la séance, résolu en français par Dumont et Séford. Lorsque ce dernier reprend la parole (34), il formule une question en anglais, ce qui lui permet à la fois d'accomplir une rupture avec ce qui précède et de s'orienter vers la langue préférée de son interlocuteur. On voit donc comment le changement de langue permet de gérer un cadre de participation complexe. A la fin de la réponse, Séford clôt la paire adjacente avec un « okay» en anglais (42) et offre la parole à Freiburg, en s'orientant à nouveau vers la langue préférée de ce site, l'anglais. De même, il s'oriente vers la langue préférée de Bruxelles (58) en offrant à ce site la possibilité de poser la dernière question. La transition vers la présentation suivante est réalisée en anglais : elle permet de rendre intelligible le passage de la discussion à l'exposé et elle projette la langue de celui-ci, qui sera effectué en anglais : dans ce cas le changement de langue permet d'accomplir localement l'intelligibilité d'un changement de type d'activité.

Les choix de langue ne sont donc déterminés ici ni par une activité particulière (comme poser des questions ou diriger la séance) ni par l'adressage à un interlocuteur particulier (p.ex. germanophone $v s$ francophone) et ne peuvent donc être expliqués par un modèle distributionnaliste ou corrélationniste général. En revanche, les choix effectués rendent reconnaissables des alignements, des contrastes ou des ruptures par rapport à l'action qui précède et des projections et orientations par rapport à celle qui va suivre, i.e. des logiques séquentielles et praxéologiques locales. Dans ce sens, ils sont le fruit de l'organisation des enchaînements tour par tour, où prennent place, de manière située, réinterprétable selon les contingences, des orientations multiples vers des préférences attribuées aux participants (participant-related CS) ou vers l'organisation de l'activité (discourse-related CS).

\subsection{Modification de la participation lors de la gestion de problèmes (techniques)}

La difficulté d'associer de manière générale et a priori un choix de langue avec un type d'activité ou de locuteur, y compris lorsque celui-ci a été localement catégorisé comme ayant une préférence linguistique forte (comme c'est le cas ici des germanophones, dont la préférence pour 1 'anglais a été exhibée par eux et co-établie par leurs partenaires), et l'intérêt d'un modèle descriptif qui soit sensible au positionnement séquentiel des choix de langue au fil du déroulement de l'interaction, est ultérieurement démontré par un dernier cas, lui aussi caractéristique des réunions étudiées ici.

Ces réunions sont en effet souvent interrompues par des problèmes techniques posés par le dispositif de la visioconférence: le problème le plus fréquent est donné par un figement de l'image à l'écran, alors que la connexion audio continue à fonctionner. La détection et la résolution de ces problèmes occasionnent des échanges où les choix de langue sont configurés autrement que lors des discussions et des présentations.

Tel est par exemple le cas lorsque, dans la même réunion que celle que nous avons analysée dans la section précédente, se manifeste un problème technique : 
(8) (TC07058 / 12.54)

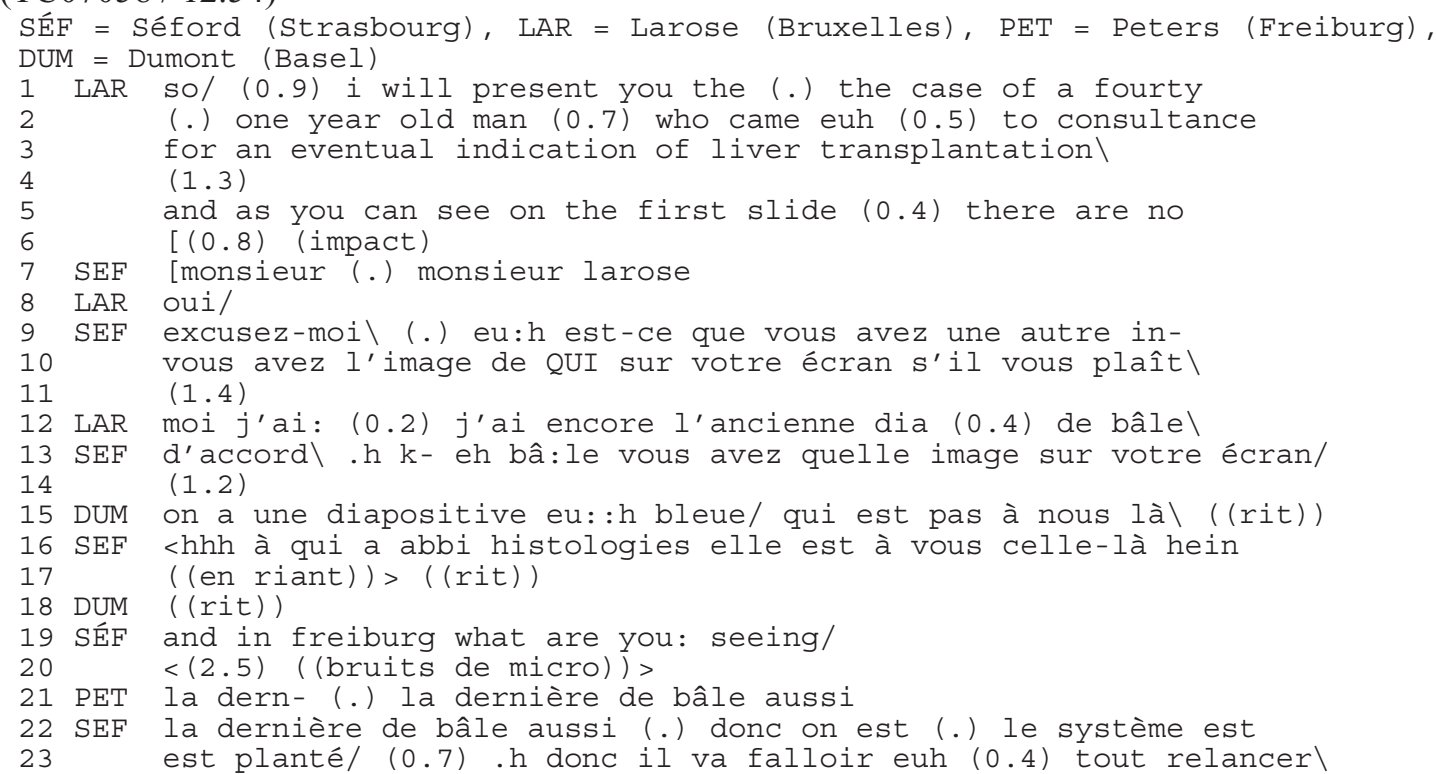

L'exposé du docteur Larose est ici interrompu par Séford suite à sa première référence à une diapositive qui ne correspond pas à celle qui est à l'écran - lequel continue à être occupé par la dernière diapositive de l'exposé précédent de Plätz portant sur la technique «abbi ». Séford initie le travail de vérification par un summons (7) en français, opérant un contraste maximal avec la langue de l'exposé et contribuant ainsi à la fois à opérer et à rendre visible l'interruption de celui-ci. Ce contraste a pour effet de changer la vision que les participants ont de la diapositive, qui ne fonctionne plus comme un support de l'exposé mais comme un élément dénotant un trouble de connexion. Le CS accomplit ici un changement de Gestalt dans l'environnement visuel de l'interaction.

Une première vérification est faite avec Larose lui-même à Bruxelles, puis avec Dumont à Bâle (13-18), enfin avec Freiburg (19). On remarquera que Séford effectue la vérification en français avec les deux premiers sites, alors qu'il initie la séquence en anglais lorsqu'il s'adresse à Freiburg, en s'orientant ainsi vers la langue préférée de ce dernier site. Toutefois, Peters répond en français : ici il ne s'aligne pas avec la préférence projetée par Séford à son encontre, mais plutôt avec la série de vérifications-confirmations qui vient d'avoir lieu avec les autres participants en français. Ainsi, de manière locale, pour la gestion du problème technique, il récuse la catégorisation de «non francophone » qu'il accepte voire revendique dans d'autres contextes; ce faisant, il manifeste ici une affiliation au groupe des participants à la visioconférence (au groupe des utilisateurs du dispositif technique en panne), en produisant une formulation qui est alignée sur les précédentes ${ }^{15}$.

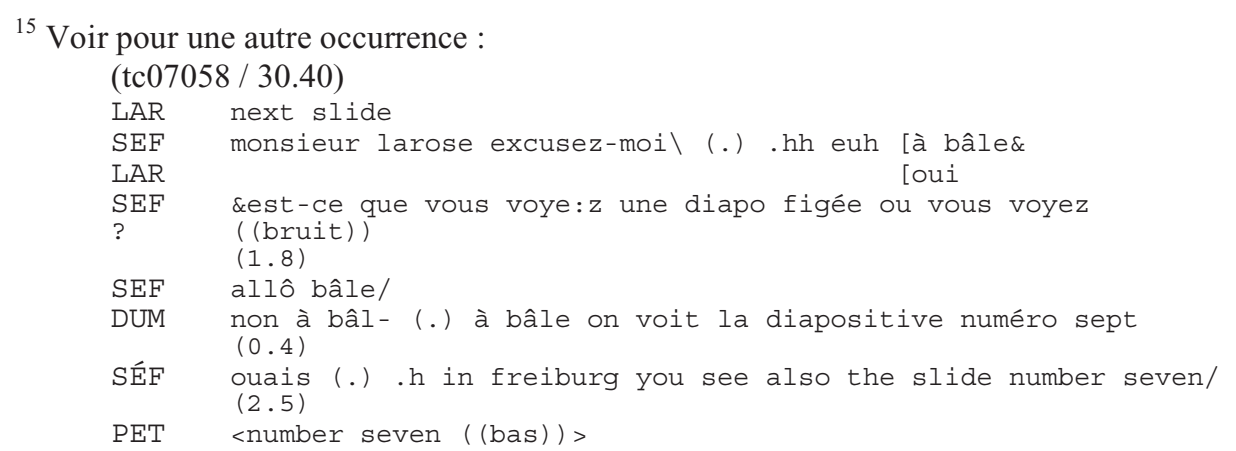


De manière intéressante, la catégorisation, la préférence linguistique, voire la compétence d'un membre est ici reconfigurée localement non seulement lors d'une activité particulière, mais aussi lors d'un enchaînement séquentiel particulier (Freiburg est adressé à la fin d'une série de vérifications, donc lorsqu'un pattern de réponse vient d'émerger et de se constituer - par rapport auquel il choisit de se positionner d'une manière plutôt que d'une autre).

\section{Conclusions}

Cet article vise une réflexion sur la manière dont les fonctionnalités pragmatiques et sociolinguistiques du CS ont été conceptualisées dans la littérature. La variété des solutions montre en retour l'intérêt de ce phénomène comme lieu de discussion et de confrontation théorique, analytique et méthodologique en linguistique.

Le cas empirique que nous avons développé permet de montrer que si le CS est effectivement sensible à des attentes, des droits et obligations, des types d'activités ou de contextes, son rendement fonctionnel ne peut être décrit de manière globale en lui attribuant une «signification sociale " a priori ni en indexant directement un ensemble de valeurs à un choix linguistique. L'indexicalité et la plasticité du CS en font une ressource qui est sensible à la configuration locale des catégories, des activités, des cadres de participation, des contextes telle qu'elle émerge du déroulement séquentiel de l'interaction: sa signification est constituée par les participants en relation étroite avec son positionnement dans l'organisation séquentielle.

Cela est le cas même lorsque les participants eux-mêmes énoncent et se donnent des règles, manifestent des orientations générales, effectuent des catégorisations, attribuent des compétences et des préférences linguistiques. Ces énoncés ont des effets configurants qui reproduisent des valeurs, des croyances, voire des idéologies et qui pèsent sur la suite des activités ; néanmoins la manière dont ces énoncés se matérialisent dans les activités ainsi que la manière dont se réalise l'organisation détaillée de ces activités relèvent toujours d'accomplissements pratiques, localement situés, prenant une forme spécifique, tour par tour, TCU par TCU, au sein de l'organisation séquentielle. C'est pourquoi, en définitive, la valeur du CS ne peut être disjointe de la position séquentielle à la quelle il se réalise. L'interprétation du CS repose donc sur le contraste que le changement de langue accomplit entre l'action qui le précède et celle qu'il introduit, entre le previous et le next, i.e. entre des positions séquentiellement ordonnées au sein d'une activité. Le fait que le CS doté d'un rendement pragmatique et interactionnel reconnaissable pour les participants opère en respectant les frontières de constituants syntaxiques ou d'autres frontières grammaticales est fonctionnel par rapport au travail organisationnel qu'il réalise, qui rend intelligibles et reconnaissables les changements de structuration des cadres de participation et des activités : là encore, cependant, la définition d'une frontière grammaticale est un accomplissement pratique

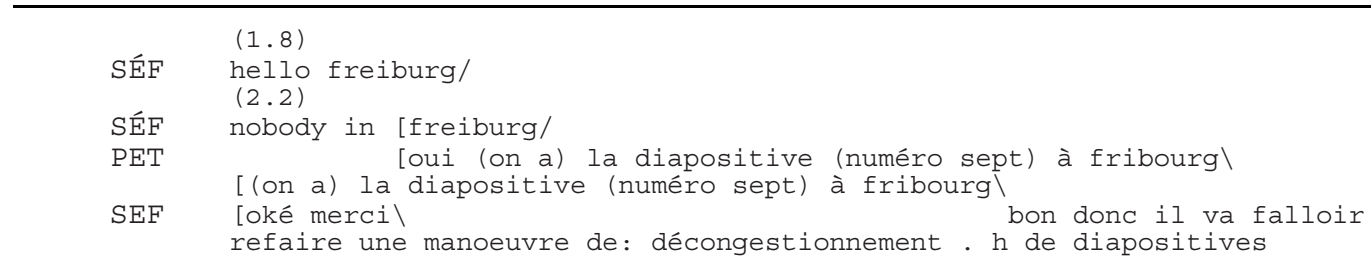

L'étude du corpus dans sa globalité montre que si Peters revendique l'anglais comme langue pour les discussions, afin de faciliter la participation de ses collègues allemands, il s'aligne avec le français dans d'autres circonstances, comme celle-ci, où les responsables des sites font face collectivement aux adversités de la technologie (de nombreux commentaires ironiques sont échangés à cette occasion à propos du fournisseur de service et constituent une opportunité pour développer des affiliations basées sur d'autres activités que l'expertise médicale). 
relevant de la manière localement située dont les participants eux-mêmes s'orientent vers ce qu'ils définissent à toutes fins pratiques comme une unité grammaticale ou linguistique.

C'est ainsi que l'approche séquentielle du CS permet de rendre compte à la fois d'une certaine stabilité des orientations des participants (vers des unités et des langues reconnaissables, vers des types d'activités, vers des formats de participation) et d'une indexicalité radicale des entités définies par ces orientations.

Conventions de transcription (valables pour les exemples $4 \mathrm{sv}$.)

Parole :

$\begin{array}{llll}{[} & \text { chevauchements } & (.) & \text { micro-pauses } \\ (2.3) & \text { pauses chronométrées } & \text { xxx } & \text { segment inaudible } \\ / \quad \backslash & \text { intonation montante/ descendante } & \text { exTRA } & \text { segment accentué } \\ ((\text { rire })) & \text { phénomènes non transcrits } & : & \text { allongement vocalique } \\ <> & \text { délimitation des phénomènes entre }(()) & \text { par- } & \text { troncation } \\ \& & \text { continuation du tour de parole } & = & \text { enchaînement rapide } \\ \wedge & \text { liaison } & . \mathrm{h} & \text { aspiration } \\ (\text { il va) } & \text { essai de transcription } & { }^{\circ} \text { bon }^{\circ} & \text { murmuré }\end{array}$

Gestes:

* * indication du début/de la fin d'un geste, décrit en italique à la ligne successive

----> continuation du geste aux lignes suivantes

(si l'auteur d'un geste n'est pas le locuteur en cours, ses initiales en minuscules sont indiquées)

Les noms des participants ont été anonymisés.

\section{Références}

Alfonzetti, Giovanna. 1998. The conversational dimension in code-switching between Italian and dialect in Sicily. Code-Switching in Conversation. Language, Interaction and Identity ed. by Peter Auer. London: Routledge.

Alvarez-Caccamo, Celso. 1990. Rethinking conversational code-switching: Codes, speech varieties, and contextualization. Proceedings of the Sixteenth Annual Meeting of the Berkeley Linguistics Society, 3-16. Berkeley: Berkeley Linguistics Society.

Alvarez-Caccamo, Celso. 1998. From 'switching code' to 'codeswitching': Toward a reconceptualisation of communicative codes. Code-Switching in Conversation. Language, Interaction and Identity ed. by Peter Auer, 27-48. London: Routledge.

Antaki, Charles \& Widdicombe, Susan M. (eds.). 1998. Identities in Talk. London: Sage.

Auer, Peter. 1984. Bilingual conversation. Amsterdam: John Benjamins.

Auer, Peter. 1988. A conversation analytic approach to code-switching. Code-switching: Anthropological and sociolinguistic perspectives ed. by Monika Heller, 187-214. Berlin: Mouton de Gruyter.

Auer, Peter. 1995. The pragmatics of code-switching: A sequential approach. One speaker two languages: Cross-disciplinary perspectives on code-switching ed. by Lesley Milroy \& Pieter Muysken, 115-135. Cambridge: Cambridge University Press. 
Auer, Peter. 1998a. From Code-switching via Language Mixing to Fused Lects: Toward a Dynamic Typology of Bilingual Speech, Interaction and Linguistic Structures 6, 1-28.

Auer, Peter. 1998b. Introduction: Bilingual conversation revisited. Code-switching in conversation: Language, interaction and identity ed. by Peter Auer, 1-24. London: Routledge.

Auer, Peter \& di Luzio, Aldo (eds.). 1992. The Contextualization of Language. Amsterdam: John Benjamins.

Auer, Peter \& Dirim, Inci. 2000. On the use of Turkish routines by adolescents of non-Turkish descent in Hamburg. Det er Conversation 501, degil mi? Perspectives on Bilinguismalism of Turkish Speaking Children and Adolescents in North Western Europe ed. by Anne Holmen \& J.Normann Jorgensen, 157-194. Copenhagen: The Danish University of Education.

Blom, Jan Petter \& Gumperz, John. 1972. The Social Meaning in Linguistic Structure: CodeSwitching in Norway. Directions in Sociolinguistics ed. by John Gumperz \& Dell Hymes, 407-34. New York: Holt, Rinehart \& Winston.

Blommaert, Jan \& Verschueren Jef. 1998. Debating Diversity: Analysing the discourse of tolerance. London : Routledge.

Bloomfield, Leonard.1933. Language. New York: Holt, Rinehart \& Winston.

Bucholtz, Mary. 1995. From mulatta to mestiza: Passing and the linguistic reshaping of ethnic identity. Gender Articulated ed. by Kira Hall \& Mary Bucholtz. New York: Routledge.

Cashman, Holly R. 2002. Constructing a Bilingual Identity: conversation analysis of Spanish/English language use in a television interview. Texas Linguistic Forum 44 (Proceedings of the Symposium About Language and Society-Austin VIII), 33-47.

Cashman, Holly R. 2005. Identities at play: language preference and group membership in bilingual talk in interaction. Journal of Pragmatics 37(3): 301-315.

Cromdal, Jakob. 2000. Code-Switching for all Practical Purposes. Bilingual Organization of Children's Play. Linköping University.

Day, Dennis. 1994. Tang's dilemma and other problems: Ethnification processes at some multicultural workplaces. Pragmatics 4/3: 315-336

Davies, Alan. 2003. The Native Speaker: Myth and Reality. Clevedon: Multilingual Matters.

Ferguson, Charles F. 1959. Diglossia. Word 15: 325-40.

Finlayson, Rosalie \& Slabbert, Sarah. 1997. 'I'll meet you halfway with language': Code-switching within a South African urban context. Language Choices ed. by Martin Pütz. John Benjamins : Amsterdam.

Fishman, Joshua A. 1965. Who speaks what language to whom and when? La Linguistique 2 : 6788.

Fishman, Joshua A. 1967. Bilingualism with and without diglossia; diglossia with and without bilingualism. Journal of Social Issues 23: 29-38. 
Garafanga, Joseph. 2001. Linguistic identities in talk-in-interaction. Journal of Pragmatics 33: 1901-1125.

Garafanga, Joseph. 2005. Demythologising language alternation studies: conversational structure vs social structure in bilingual interaction. Journal of Pragmatics 37: 281-300.

Garfinkel, Harold. 1967. Studies in Ethnomethodology. Englewood Cliffs : Prentice Hall.

Goodwin, Marjorie H. 1999. Constructing Opposition with Girls' Games. Reinventing Identities: The Gendered Self in Discourse ed. by Mary Bucholtz, A.C. Liang \& Laurel Sutton, 388409. New York-Oxford: Oxford University Press.

Goodwin, Marjorie H. 2003. The Relevance of Ethnicity, Class, and Gender in Children's Peer Negotiations. The Handbook of Language and Gender ed. by Janet Holmes \& Miriam Meyerhoff, 229-251. Malden, MA/Oxford: Blackwell.

Grosjean, François. 1982. Life with Two Languages. Cambridge: Harvard University Press.

Gumperz, John J. 1982. Discourse Strategies. Cambridge: Cambridge University Press.

Haugen, Einar. 1956. Bilingualism in the Americas: A Bibliography and Research Guide. University of Alabama Press.

Heller, Monica. 1982. Negotiations of language choice in Montreal. Language and social identity ed. by John J. Gumperz, 108-118. Cambridge : Cambridge University Press.

Heller, Monica. 1992. The politics of codeswitching and language choice. Journal of Multilingual and Multicultural Development 13(1): 123-142.

Heller, Monica. 1995. Language Choice, Social Institutions, and Symbolic Domination. Language in Society 24(3): 373-406.

Hester, Stephen \& Eglin, Peter. 1997. Culture in Action. Studies in Membership Categorization Analysis. Washington: University Press of America.

Hewitt, Roger. 1986. White Talk Black Talk. Inter-racial friendship and communication amongst adolescents. Cambridge: Cambridge University Press.

Irvine, Judith. 1987. Domains of description in the ethnography of speaking : A retrospective on the 'speech community'. Performance, Speech Community and Genre ed. by Richard Bauman, Judith Irvine \& Susan U. Philips, 13-24. Chicago: Centre for Psychosocial Studies.

Kandiah, Thiru. 1998. Epiphanies of the deathless native user's manifold avatars: a post-colonial perspective on the native speaker. The Native Speaker: multilingual perspectives ed. by Rajendra Singh, 79-110. New Delhi: Sage.

Le Page, Robert B. \& Tabouret-Keller, Andrée. 1985. Acts of Identity: Creole-based approaches to language and ethnicity. Cambridge: Cambridge University Press.

Li Wei. 2002. 'What do you want me to say?' On the Conversation Analysis approach to bilingual interaction. Language in Society 31(2): 159-180.

Li Wei. 2005. "How can you tell?" Towards a common sense explanation of conversational codeswitching. Journal of Pragmatics 37(3): 375-389. 
Lüdi, Georges \& Py, Bernard. 2003 [1984]. Etre bilingue, Bern : Peter Lang.

Meeuwis, Michael \& Blommaert, Jan. (1994). The Markedness Model and the absence of society: Remarks on codeswitching. Multilingua 13(4): 387-423.

Mondada, Lorenza. 1999. L'accomplissement de l'"étrangéité" dans et par l'interaction: procédures de catégorisation des locuteurs. Langages $134: 20-34$.

Mondada, Lorenza. 2000a. Les effets théoriques des pratiques de transcription. Revue des linguistes de l'université Paris X (LINX) 42 : 131-150.

Mondada, Lorenza. 2000b. La compétence de catégorisation: procédés situés de catégorisation des ressources linguistiques. La notion de contact de langues en didactique éd. par Pierre Martinez \& S. Pekarek Doehler, 81-102. Paris: ENS Editions \& Didier Erudition.

Mondada, Lorenza. 2002. Pratiques de transcription et effets de catégorisation. Cahiers de Praxématique 39 : 45-75.

Mondada, Lorenza. 2004. Ways of 'Doing Being Plurilingual' In International Work Meetings. Second Language Conversations ed. by Rod Gardner \& Johannes Wagner, 27-60. London: Continuum.

Mondada, Lorenza. 2006. La pertinenza del dettaglio : registrazione e trascrizione di dati video per la linguistica interazionale. Trascrivere la lingua. Dalla filologia all'analisi conversazionale ed. by Yvette Bürki \& Elwys de Stefani, 313-344. Bern : Peter Lang.

Mondada, Lorenza. (in press). Bilingualism and the analysis of talk at work: Code-switching as a resource for the organization of action and interaction. Bilingualism. Advances in Linguistics ed. by Monika Heller, Macmillan.

Myers-Scotton, Carol. 1988. Codeswitching as indexical of social negotiation. Codeswitching: Anthropological and Sociolinguistic Perspectives ed. by Monika Heller. Berlin: Mouton de Gruyter.

Myers-Scotton, Carol. 1993a. Common and uncommon ground: social and structural factors in codeswitching. Language in Society 22: 475-503.

Myers-Scotton, Carol. 1993b. Social Motivations for Codeswitching: Evidence from Africa. Oxford: Clarendon Press.

Myers-Scotton, Carol \& Bolonyai, Agnes. 2001. Calculating speakers: Codeswitching in a rational choice model. Language in Society 30(1): 1-28.

Orletti, Franca \& Testa, Renata. 1991. La trascrizione di un corpus di interlingua. Studi Italiani di Linguistica Teorica e Applicata 20(2): 243-283.

Poplack, Shana 1980. Sometimes I'll start a sentence in English y TERMINO EN ESPAÑOL. Linguistics 18: 581-616.

Rampton, Ben. 1995. Crossing: language and ethnicity among adolescents. London: Longman.

Renaud Patrick 1998. Absoute pour un locuteur natif. Le Français en Afrique : Francophonies, 12, éd. par Ambroise Queffelec, 257-72. 
Renaud Patrick. 2005. Domain and Role Specific Research. Handbook of Sociolinguistics ed. by Ulrich Ammon \& al., 1447-1459. Berlin: Mouton de Gruyter.

Sacks, Harvey. 1972. An initial investigation of the usability of conversational materials for doing sociology. Studies in Social Interaction ed. by David Sudnow, 31-74. New York: Free Press.

Sacks, Harvey. 1992. Lectures on Conversation. London: Blackwell, 2 vols.

Schegloff, Emanual A. 1972. Sequencing in conversational openings. Directions in Sociolinguistics: the Ethnography of Communication ed. by John J. Gumperz \& Dell Hymes, 346-380. New York: Holt, Rinehart \& Winston.

Schegloff, Emanual A. 1992. In another context. Rethinking Context: Language as an Interactive Phenomenon ed. by Alessandro Duranti \& Charles Goodwin, 191-227. Cambridge: Cambridge University Press.

Sebba, Mark \& Wootton, Tony. 1998. We, They and Identity: Sequential vs. Identity-related Explanation in Code-switching. Code-switching in conversation ed. by Peter Auer, 262289. London: Routledge.

Sobrero, Alberto. 1992. Alternanza di codici fra italiano e dialetto : dalla parte del parlante. Il dialetto nella conversazione. Ricerche di dialettologia pragmatica ed. by Alberto A. Sobrero, 11-30. Galatina : Congedo Editore.

Steensig, Jakob. 2003. Conversation analysis and the study of bilingual interaction. Nordlyd 31/5: 796-818.

Torras, Maria C. \& Garafanga, Joseph. 2002. Social identities and language alternation in nonformal institutional bilingual talk: Trilingual service encounters in Barcelona. Language in Society 31: 527-548.

Turner, Victor. 1982. Liminal to liminoid in play, flow and ritual. From Ritual to Theatre. 20-60. New York: Performing Arts Journal Publications.

Weinreich, Uriel. 1968. Language in Contact: Findings and problems. The Hague: Mouton.

Zimmerman, Don. 1992. Achieving context : Openings in emergency calls. Text in Context ed. by Graham Watson \& Robert M. Seiler. Newbury Park: Sage. 